Nonlin. Processes Geophys., 20, 899-919, 2013

www.nonlin-processes-geophys.net/20/899/2013/

doi:10.5194/npg-20-899-2013

(c) Author(s) 2013. CC Attribution 3.0 License.

(c) (i)

\title{
Ion motion in the current sheet with sheared magnetic field - Part 2: Non-adiabatic effects
}

\author{
A. V. Artemyev ${ }^{1,2}$, A. I. Neishtadt ${ }^{1,3}$, and L. M. Zelenyi ${ }^{1}$ \\ ${ }^{1}$ Space Research Institute RAS, Profsouznaya st., 84/32, GSP-7, 117997 Moscow, Russia \\ ${ }^{2}$ Laboratoire de physique et chimie de l'environnement et de l'Espace (LPC2E), UMR7328, CNRS - University of Orleans, \\ Orleans, France \\ ${ }^{3}$ Department of Mathematical Sciences, Loughborough University, Loughborough LE11 3TU, UK
}

Correspondence to: A. V. Artemyev (ante0226@gmail.com)

Received: 4 July 2013 - Revised: 12 September 2013 - Accepted: 20 September 2013 - Published: 31 October 2013

\begin{abstract}
We investigate dynamics of charged particles in current sheets with the sheared magnetic field. In our previous paper (Artemyev et al., 2013) we studied the particle motion in such magnetic field configurations on the basis of the quasi-adiabatic theory and conservation of the quasiadiabatic invariant. In this paper we concentrate on violation of the adiabaticity due to jumps of this invariant and the corresponding effects of stochastization of a particle motion. We compare effects of geometrical and dynamical jumps, which occur due to the presence of the separatrix in the phase plane of charged particle motion. We show that due to the presence of the magnetic field shear, the average value of dynamical jumps is not equal to zero. This effect results in the decrease of the time interval necessary for stochastization of trapped particle motion. We investigate also the effect of the magnetic field shear on transient trajectories, which cross the current sheet boundaries. Presence of the magnetic field shear leads to the asymmetry of reflection and transition of particles in the current sheet. We discuss the possible influence of single-particle effects revealed in this paper on the current sheet structure and dynamics.
\end{abstract}

\section{Introduction}

Current sheets (CSs) represent one of the most important and intriguing plasma objects in space plasmas. CSs have been studied and observed in planetary magnetospheres (Baumjohann et al., 2007; Artemyev and Zelenyi, 2013) and the solar corona (see Syrovatskii, 1979; Parker, 1994, and references therein). Theory of formation and stability of CSs is based on the detailed description of a charged particle motion (see Whipple et al., 1984; Sitnov et al., 2000; Zelenyi et al., 2000, 2011). The motion of charged particles in inhomogeneous magnetic fields of CSs can be described analytically in two different classes of systems. When a spatial scale of the magnetic field inhomogeneity is much larger than a particle's Larmor radius, the classical theory of the guiding-centre motion can be applied (Northrop, 1963; Sivukhin, 1965). Another class contains systems where a spatial scale of the magnetic field inhomogeneity is much smaller than a particle's Larmor radius. In this case the so-called theory of the quasi-adiabatic motion is used (Büchner and Zelenyi, 1986; Büchner and Zelenyi, 1989; Chen, 1992; Vainchtein et al., 2005; Zelenyi et al., 2013).

Both theories are based on the conservation of the additional approximate invariant of motion - invariant of action (Landau and Lifshitz, 1960). For the guiding-centre motion, the magnetic moment is an adiabatic invariant, while in the quasi-adiabatic theory, an analogue of the magnetic moment is called a quasi-adiabatic invariant (Büchner and Zelenyi, 1989). Violations of invariant conservation are called jumps of the adiabatic (or quasi-adiabatic) invariant. For the guiding-centre theory the expressions for jumps of the magnetic moment and effects of these jumps are described in detail in several comprehensive papers (see Birmingham, 1984; Chirikov, 1987; Büchner and Zelenyi, 1989; Delcourt et al., 1994, and references therein). The expression for jumps of the quasi-adiabatic invariant is derived for systems with a small scale of the magnetic field inhomogeneity (Neishtadt, 1986; Cary et al., 1986; Neishtadt, 1987; Büchner and Zelenyi, 1989). In contrast with the 
guiding-centre theory, here violation of the adiabaticity corresponds to change of a type of particle motion (Timofeev, 1978). As a result, we deal with the systems containing the separatrix in the phase plane. The separatrix demarcates the regions of different types of a particle motion. Crossings of this separatrix result in jumps of the quasi-adiabatic invariant.

Effects of jumps of the quasi-adiabatic invariant are important not only for dynamics of an individual particle but also for dynamics and evolution of the entire CS configuration (see Zelenyi et al., 2002a, 2003). Moreover, destruction of adiabaticity results in stochastization of particle motion (Chen and Palmadesso, 1986; Büchner and Zelenyi, 1989; Burkhart and Chen, 1991; Büchner, 1991; Burkhart et al., 1995). These effects are important for particle acceleration in the CSs of the solar corona (Litvinenko, 2003; Anastasiadis et al., 2008) and in planetary magnetotails (Büchner and Zelenyi, 1990; Ashour-Abdalla et al., 1992; Cheng and Decker, 1992; Ashour-Abdalla et al., 1994; Delcourt et al., 2003; Grigorenko et al., 2011; Zhou et al., 2012; Zelenyi et al., 2013). Isotropization of particle velocity distribution due to stochastization of particle motion influences the CS configuration in the vicinity of the reconnection region (Le et al., 2013). Moreover, the same effect of the destruction of quasi-adiabatic invariants can play a significant role in particle transport in radiation belts (see Ukhorskiy et al., 2011, and references therein) and in dynamics of the laboratory plasma (e.g. Chirikov, 1979; Carati et al., 2012).

For the simple model of CS without a shear component of the magnetic field, $B_{y}$ (here and in the following we use the GSM coordinate system), the theory of jumps of the quasi-adiabatic invariant is described in details by Büchner and Zelenyi (1989). However, the $B_{y}$ component is often present in the Earth's magnetotail (see Petrukovich, 2011, and references therein) and in CSs of the solar corona (e.g. Masuda et al., 2001; Schrijver, 2009). This component can affect particle acceleration (Litvinenko, 1996), CS structure (Whipple et al., 1984; Roth et al., 1996; Artemyev, 2011; Malova et al., 2012) and CS dynamics (Galeev et al., 1986; Kuznetsova and Roth, 1995; Silin and Büchner, 2003; Karimabadi et al., 2005; Artemyev and Zimovets, 2012). The influence of $B_{y}$ on particle motion has been studied in several papers with the help of numerical modelling of test trajectories (Karimabadi et al., 1990; Büchner and Zelenyi, 1991; Zhu and Parks, 1993; Baek et al., 1995; Chapman and Rowlands, 1998; Delcourt et al., 2000). It was noticed that $B_{y}$ dramatically influences particle scattering due to jumps of the quasi-adiabatic invariant (Büchner and Zelenyi, 1991; Kaufmann et al., 1994; Holland et al., 1996). However, no analytical theory of this scattering has been developed so far.

In our previous paper we presented the analytical theory of the quasi-adiabatic particle motion in the CS with an arbitrary value of $B_{y}$ (Artemyev et al., 2013). It was shown that dynamics of particles in the CS with $B_{y} \neq 0$ are substantially more complicated than particle dynamics in non-sheared magnetic field configurations. Therefore, several conclusions considered earlier for CSs without $B_{y}$ should be revised for CSs with $B_{y} \neq 0$.

The process of a particle interaction with the CS can be represented as a combination of scattering, reflection from the CS and transition through the CS. These three phenomena define efficiency of the ion acceleration (see review by Grigorenko et al., 2011), determine amplitudes of ion electric currents, which, in fact, support the CS configuration (see review by Artemyev and Zelenyi, 2013), and are responsible for various aurora manifestations in the Earth's polar magnetosphere (see review by Paschmann et al., 2002).

The first part of the present paper is devoted to $B_{y}$ influence on particle interaction with the CS. We consider reflection and transition of particles coming to the CS from the CS boundaries. The influence of $B_{y}$ on particle scattering in the CS and corresponding stochastization of particle motion is studied in the second part of the present paper, where we concentrate on the investigation of the violation of the adiabaticity.

\section{General equations}

We study dynamics of particles in the plasma configuration with the sheared magnetic field reversal. This configuration can be represented by the system with the magnetic field $\boldsymbol{B}=B_{x}(z) \boldsymbol{e}_{x}+B_{y} \mathbf{e}_{y}+B_{z} \boldsymbol{e}_{z}$, where $B_{z}>0$ and $B_{y}>0$ are constant. Such a model allows also for the CS boundaries to be taken into account: $B_{x}=B_{0}(z / L)$ at $|z / L|<1$ and $B_{x}=$ $\pm B_{0}$ at $|z / L|>1$. In the CS central region (where $|z / L|<1$ ) the vector potential is $\boldsymbol{A}=B_{y} z \boldsymbol{e}_{x}+\left(B_{z} x-B_{0} z^{2} / 2 L\right) \boldsymbol{e}_{y}$. The system is homogeneous along the $y$ axis, and the corresponding canonical momentum, $p_{y}$, is conserved. Thus, we can shift the coordinate system along $x$ to set $p_{y}=0$. Due to stationarity of the magnetic field the energy of each particle is conserved, $h=$ const. The Hamiltonian of a particle with mass $m$ and charge $q$ in this system has the form

$H=\frac{1}{2} p_{z}^{2}+\frac{1}{2}\left(p_{x}-s z\right)^{2}+\frac{1}{2}\left(\kappa x-\frac{1}{2} z^{2}\right)^{2}$,

where we use dimensionless variables and parameters: $H \rightarrow$ $H / 2 h, \boldsymbol{p} \rightarrow \boldsymbol{p} / \sqrt{2 h m}, \boldsymbol{r} \rightarrow \boldsymbol{r} / \sqrt{\rho_{0} L}$, dimensionless time $t \rightarrow t \sqrt{2 h /\left(\rho_{0} L m\right)}$, and parameters $\kappa=\left(B_{z} / B_{0}\right) \sqrt{L / \rho_{0}}$ and $s=\left(B_{y} / B_{0}\right) \sqrt{L / \rho_{0}}\left(\rho_{0}=\sqrt{2 h m} c /\left(q B_{0}\right)\right.$ is a Larmor radius). This normalization corresponds to particle motion in the 3-D energy level $H\left(z, p_{z}, \kappa x, p_{x}\right)=1 / 2$ of 4-D phase space $\left(z, p_{z}, \kappa x, p_{x}\right)$. We assume that the parameter $\kappa$ is small $(\kappa \in[0.01,0.1]$ for energetic and thermal ions in the Earth's magnetotail CS (see review by Zelenyi et al., 2011, and references therein)). Therefore, variables $\left(\kappa x, p_{x}\right)$ could be considered as slow ones, and variables $\left(z, p_{z}\right)$ as fast. In this paper we consider only systems with $s>\left(\pi^{-1} \ln 2\right) \kappa$ when effects of non-zero $B_{y}$ are well distinguished. For systems with $s<\left(\pi^{-1} \ln 2\right) \kappa$, one cannot separate effects of $B_{y}$ 
and effects of particle scattering due to dynamical jumps of the quasi-adiabatic invariant (see Artemyev et al., 2013).

Here we discuss general features of the particle dynamics described by Hamiltonian (1). An example of the particle trajectory is shown in Fig. 1 (top-left panel). One can see that the $z$ coordinate oscillates fast, while coordinates $\kappa x$ and $\kappa y$ change slowly. This is an effect of smallness of the parameter $\kappa$. Therefore, we can describe system (1) using the separation of the particle motion into fast oscillations in the plane $\left(z, p_{z}\right)$ and slow evolution of coordinates $\left(\kappa x, p_{x}\right)$. At each point in the plane $\left(\kappa x, p_{x}\right)$ the particle oscillates in the effective potential $U(z)=\frac{1}{2}\left(p_{x}-s z\right)^{2}+\frac{1}{2}\left(\kappa x-\frac{1}{2} z^{2}\right)^{2}$ at the energy level $H=1 / 2$ (see details in Artemyev et al., 2013). The corresponding trajectory in the plane $\left(z, p_{z}\right)$ can be located inside the separatrix loops (e.g. Fig. 1a) or outside the separatrix loops (e.g. Fig. 1e). Motion inside the separatrix loops corresponds to particle oscillations in one of the two small potential wells described by potential $U(z)$. Motion outside the separatrix loops corresponds to either (1) merging of the two small potential wells into a single well, and particle oscillations within this newly formed well; or to (2) oscillations above the two small wells in the potential $U(z)$ (see details in Artemyev et al., 2013). When the particle crosses the separatrix in the plane $\left(z, p_{z}\right)$, it crosses the uncertainty curve (Wisdom, 1985) in the plane $\left(\kappa x, p_{x}\right)$ (this curve is shown as a black solid curve in Fig. 1, top-right panel).

We start the description of the particle trajectory at the point (A). At this point the particle oscillates inside the right potential well far from the separatrix (Fig. 1a) and moves along the field line towards the neutral plane $z=0$. At the point $(\mathrm{F})$ the particle trajectory in the $\left(z, p_{z}\right)$ plane approaches the separatrix (Fig. 1f). Then the particle crosses the separatrix and comes to the point $(\mathrm{E})$. At this point the particle oscillates across the neutral plane, i.e. the trajectory in the $\left(z, p_{z}\right)$ plane crosses $z=0$ (Fig. 1e). Then the particle crosses the separatrix again and becomes trapped in the left potential well (Fig. 1b and c). Inside this well the particle approaches the neutral plane without crossing of the uncertainty curve (Fig. 1d). We emphasize that the particle turns over the uncertainty curve and comes to the neutral plane without crossing of the separatrix. Comparison of panels (d) and (e) shows the difference of two fragments of the particle trajectory. If the particle crosses the separatrix, it starts oscillating across the neutral plane with about double amplitude of $z$. If the particle comes to the neutral plane $z=0$ without crossing the separatrix, it continues oscillating across the field line, which crosses the neutral plane. As a result, amplitude of particle oscillations is approximately two times smaller in the second case (compare panels (e) and (d)).

At fixed values $\left(\kappa x, p_{x}\right)$ the trajectory in the $\left(z, p_{z}\right)$ plane is closed, i.e. motion in the plane $\left(z, p_{z}\right)$ is periodic (see Fig. 1a-f). Thus, we can introduce the invariant of motion $I_{z}=(1 / 2 \pi) \oint p_{z} d z$ (Landau and Lifshitz, 1960). This invariant is often called quasi-adiabatic, in contrast to the classical adiabatic invariant represented by the magnetic moment
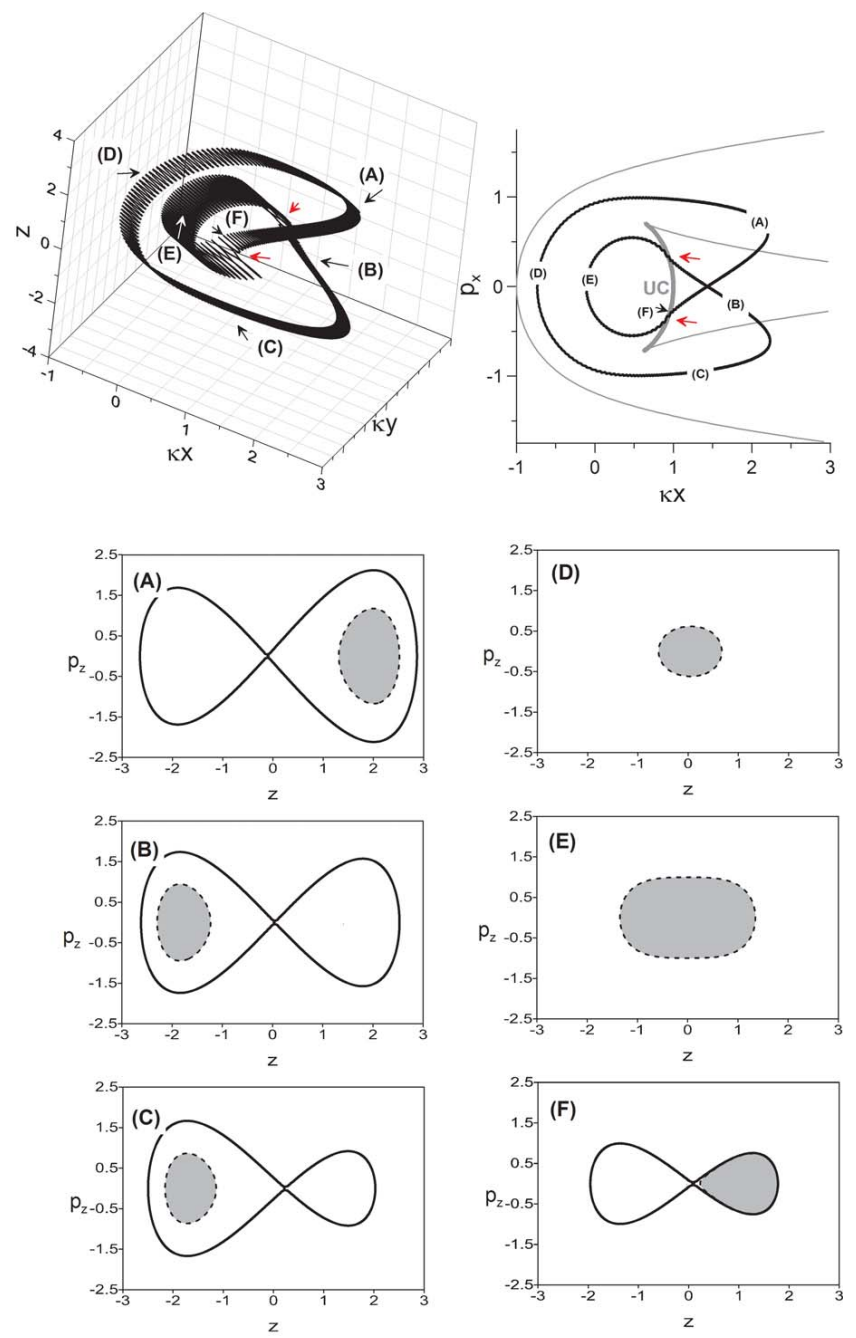

Fig. 1. The particle trajectory in 3-D space and its projection onto the plane $\left(\kappa x, p_{x}\right)$ are shown in top panels. Red arrows show positions of the uncertainty curve (UC) crossings. The corresponding fragments of the particle trajectory in the plane $\left(z, p_{z}\right)$ are presented in the bottom panels. The solid curve is the separatrix, while the dashed curve is the trajectory. Grey colour indicates the area, which is equal to $2 \pi I_{z}$.

(Büchner and Zelenyi, 1986; Büchner and Zelenyi, 1989). Conservation of this invariant is provided by the separation of timescales of evolution of $z$ and $\kappa x$. For constant energy $(H=1 / 2)$ the equation $I_{z}\left(\kappa x, p_{x}\right)=$ const describes particle trajectories in the plane $\left(\kappa x, p_{x}\right)$ (Büchner and Zelenyi, 1986). However, this equation can be violated when particle trajectories cross the separatrix in the plane $\left(z, p_{z}\right)$ (Neishtadt, 1986; Cary et al., 1986; Neishtadt, 1987). For example, let far from the neutral plane, $z=0$ particles oscillate inside one of the two admissible potential wells. When particles approach the neutral plane they can change their mode of motion from the oscillations in one well to the oscillations in the other well. This change corresponds to crossing 
of the uncertainty curve in the plane $\left(\kappa x, p_{x}\right)$. As a result, the value of $I_{z}$ experiences the jump. This jump consists of two parts: (1) the geometrical jump corresponding to the difference of the areas surrounded by trajectories before and after separatrix crossings, and (2) the dynamical jump corresponding to the singularity of the period of $z$ oscillations in the vicinity of the separatrix (Timofeev, 1978; Neishtadt, 1986; Cary et al., 1986; Neishtadt, 1987). The dynamical jump has a small $(\sim \kappa \ln \kappa)$ value, which depends on coordinates of the separatrix crossing in the planes $\left(z, p_{z}\right)$ and $\left(\kappa x, p_{x}\right)$. A position of crossing of the uncertainty curve in the plane $\left(\kappa x, p_{x}\right)$ is well defined by the equation $I_{z}\left(\kappa x, p_{x}\right)=$ const, while a position of crossing of the separatrix in the plane $\left(z, p_{z}\right)$ can be considered as random because the oscillations of $\left(z, p_{z}\right)$ are fast (i.e. even a small $\sim \kappa$ change of initial coordinates in the plane $\left(z, p_{z}\right)$ can change the position of separatrix crossing and the value of the dynamical jump very substantially). Thus, the dynamical jump is assumed to be a quasi-random variable. The geometrical jump does not depend on $\kappa$, and is well prescribed (it depends only on position of crossing of the uncertainty curve in the plane $\left.\left(\kappa x, p_{x}\right)\right)$. Particle dynamics with effects of the geometrical jumps were considered by Artemyev et al. (2013). In the present paper (in Sect. 4) we describe effects of dynamical jumps and corresponding violation of the adiabaticity of particle motion.

In the previous paper (Artemyev et al., 2013) we considered the system with Hamiltonian (1). However, the presence of the CS boundaries was not taken into account. It is appropriate as particle trajectories do not cross the planes $z= \pm \lambda$, where $\lambda=\sqrt{L / \rho_{0}}$. In the present paper (in Sect. 3) we consider effects of the boundaries on the particle motion.

It was shown in Artemyev et al. (2013) that, if $s \neq 0$, the particle trajectories can have two options for possible prolongations in the plane $\left(\kappa x, p_{x}\right)$ after crossing of the uncertainty curve. Thus, there is a splitting of the adiabatic $\left(I_{z}=\right.$ const $)$ trajectories with certain probabilities of various prolongations. We describe details of this effect in Sect. 5.

\section{The boundaries of the current sheet}

The CS boundaries are located at $z= \pm L$ (in the dimensionless variables $z= \pm \lambda$, where $\lambda=\sqrt{L / \rho_{0}}$ ). Beyond these boundaries $(|z|>\lambda)$ the value of the $B_{x}$ component of the magnetic field is constant, $B_{x}= \pm B_{0}$. Thus, once particles reach these boundaries, they escape from the CS. In this section we describe particle motion in the CS including the boundaries. We assume that the crossing of the boundaries $|z|=\lambda$ occurs when a particle moving from the region $|z|<\lambda$ crosses them. A particle crosses the boundaries when its trajectory (calculated at fixed $\left.\left(\kappa x, p_{x}\right)\right)$ in the $\left(z, p_{z}\right)$ plane is located at the space domain $|z|>\lambda$ entirely. Thus, we do not take into account situations when only a fragment of a trajectory is located in the domain $|z|>\lambda$. In this consideration we also neglect small random (dynamical) jumps of $I_{z}$, i.e. we suppose that $\kappa$ is small enough and describe particle motion at time interval $\sim 1 / \kappa$.

We consider three scenarios of particle motion. The first scenario corresponds to particles starting from the $z=\lambda$ boundary. These particles can be reflected from the CS or transit through the CS. In the case of transition, particles reach the opposite boundary $z=-\lambda$. Reflected particles can reach the initial boundary $z=\lambda$ or become trapped in the CS (in this case their reflection points should be below the boundaries). The second scenario corresponds to particles starting at the boundary $z=-\lambda$. These particles cannot be reflected from the CS and reach the opposite boundary $z=\lambda$. The third scenario corresponds to particles initially trapped in the CS. We show how the positions of reflection points of these particles can be changed due to geometrical jumps of $I_{z}$, resulting in escape of particles from the CS. These are three typical scenarios, i.e. each complex particle trajectory can be considered as a combination of trajectories described by these scenarios.

All these scenarios are characteristic for trajectories in the system with $s<0.35$. For $s \in(0.35,1)$ the reflection from the CS is impossible: all particles from the upper boundary $z=\lambda$ pass through the CS (with or without a half-rotation around $B_{z}$ ) and all particles from the bottom boundary $z=$ $-\lambda$ cross the CS without making turns around $B_{z}$ (see description of such trajectories in Artemyev et al., 2013).

The general description of particle motion in the system without dynamical jumps of $I_{z}$ is based on consideration of adiabatic trajectories in the plane $\left(\kappa x, p_{x}\right)$. These trajectories are determined by the equation $I_{z}\left(\kappa x, p_{x}\right)=$ const. Due to conservation of the particle energy, each point in the plane $\left(\kappa x, p_{x}\right)$ corresponds to a certain closed trajectory in the plane $\left(z, p_{z}\right)$. As a result, if we know all possible particle positions in the plane $\left(\kappa x, p_{x}\right)$ for a given value of $I_{z}$, we can predict whether this particle crosses the boundaries.

\subsection{The first scenario}

We consider a particle that approaches the neutral plane being within the right one of the two small potential wells. We can introduce two quasi-adiabatic invariants (calculated for the right and the left wells):

$$
\left\{\begin{array}{l}
I_{z, r, l}=\pi \int_{z_{-r, l}}^{-1} \sqrt[z_{+r, l}]{2 H-\left(p_{x}-s z\right)^{2}-\left(\kappa x-\frac{1}{2} z^{2}\right)^{2}} \mathrm{~d} z \\
\left(p_{x}-s z_{ \pm r, l}\right)^{2}+\left(\kappa x-\frac{1}{2} z_{ \pm r, l}^{2}\right)^{2}=2 H
\end{array} .\right.
$$

Here one can take into account that $H=1 / 2$ due to the normalization used. The CS boundaries $z= \pm \lambda$ correspond to the certain values $\left(\kappa \bar{x}, \bar{p}_{x}\right)$ of slow variables

$$
\left\{\begin{array}{l}
2 H=\left(\bar{p}_{x}-s \lambda\right)^{2}+\left(\kappa \bar{x}-\frac{1}{2} \lambda^{2}\right)^{2} . \\
I_{z, r}=I_{z, r}\left(\kappa \bar{x}, \bar{p}_{x}\right)
\end{array} .\right.
$$




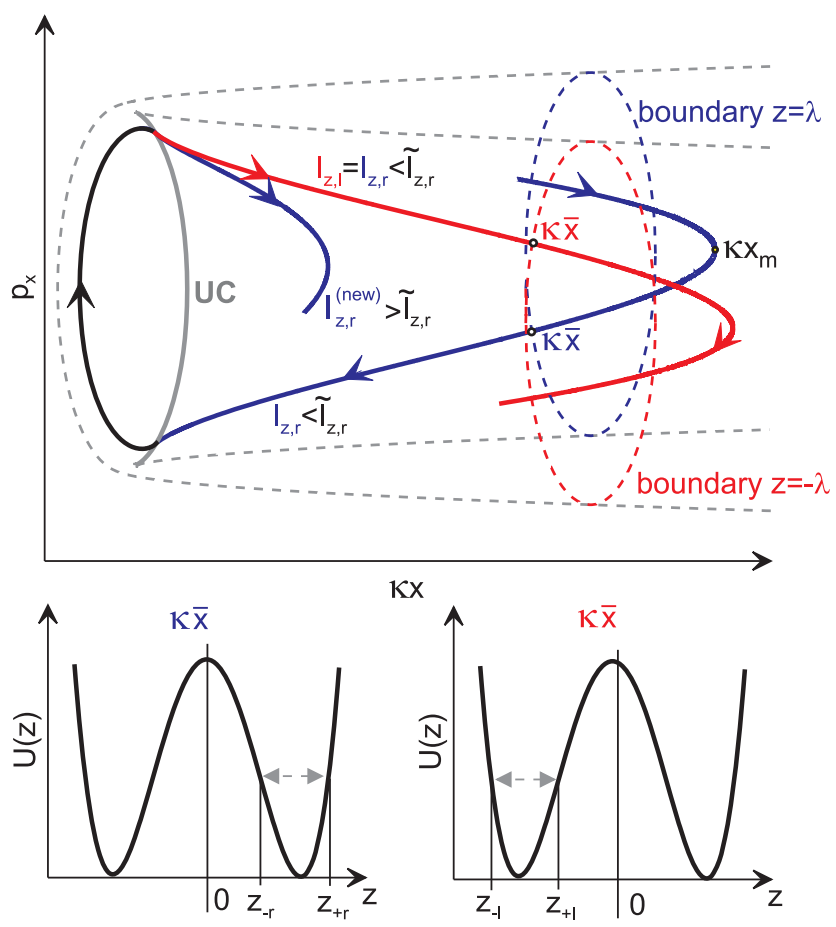

Fig. 2. Schematic view of particle trajectories before and after the neutral plane crossing. The uncertainty curve (UC) is shown by the grey solid line. Bottom panels show profiles of potential energy $U(z)$ and particle positions for two points in the plane $\left(\kappa x, p_{x}\right)$

For positive and negative values of $\pm \lambda$ this system can have four, three, two and one solutions, or can even have no solutions. The existence of four solutions means that the corresponding particle crosses the boundaries with $p_{z}=0$ two times during the motion away from the CS (with the increase of $z$ ), and crosses the boundaries with $p_{z}=0$ two times during the motion towards the neutral plane. In the first case the particle should leave the CS, because its trajectory in the plane $\left(z, p_{z}\right)$ is above one of the boundaries $|z|=\lambda$. Existence of two, three and one solutions of system (2) corresponds to particles "partially" crossing (or touching) of the boundaries in the plane $\left(z, p_{z}\right)$. In these cases we assume that particles remain in the CS. When the system (2) does not have solutions, the corresponding particle does not cross the boundaries (its reflection points are located in the region within the boundaries).

For a given value of $I_{z}$ we introduce $\kappa x_{m}$ as the most distant point in the plane $\left(\kappa x, p_{x}\right)$ that can be approached by the corresponding trajectory. Comparison of $\kappa x_{m}$ and $\kappa \bar{x}$ shows whether the particle crosses the boundaries $\left(\kappa x_{m}>\kappa \bar{x}\right)$ or not $\left(\kappa x_{m}<\kappa \bar{x}\right)$. In the plane $\left(\kappa x, p_{x}\right)$ a smaller value of $I_{z}$ corresponds to a larger value of $\kappa x_{m}$. Thus, there is a certain value of $I_{z}$ corresponding to $\kappa \bar{x}=\kappa x_{m}$ (we denote this value as $\tilde{I}_{z}$ ). All particles with $I_{z}<\tilde{I}_{z}$ cross the boundaries, while particles with $I_{z}>\tilde{I}_{z}$ cannot cross the boundaries. The equation for the most distant point $\kappa x_{m}$ at a given value of $I_{z}$ can
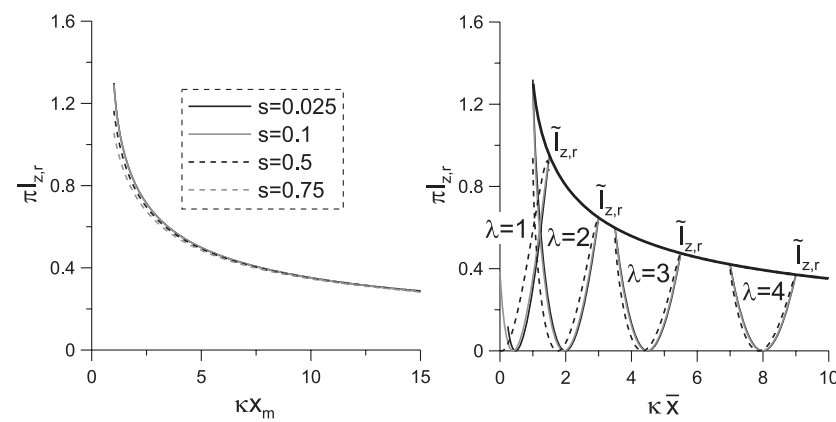

Fig. 3. Left panel: the dependence of $\kappa x_{m}$ on $I_{z}$ for various values of $s$. Right panel: $I_{z}$ as a function of $\kappa \bar{x}$ from the left panel and corresponding dependencies for various $\lambda$.

be written as

$\left\{\begin{array}{l}\frac{\partial I_{z}}{\partial p_{x}}=\oint \frac{\left(p_{x m}-s z\right) \mathrm{d} z}{\sqrt{2 H-\left(p_{x m}-s z\right)^{2}-\left(\kappa x_{m}-\frac{1}{2} z^{2}\right)^{2}}}=0 \\ I_{z, r}=I_{z, r}\left(\kappa x_{m}, p_{x m}\right)\end{array}\right.$.

In the case with $s=0$ we have $p_{x m}=0$ (as it was obtained by Büchner and Zelenyi, 1989). The solution of this system is shown in Fig. 3 (left panel). One can see that $\kappa x_{m}$ depends on $s$ only slightly. The general form of this dependence is close to the one obtained from the asymptote of $I_{z}:\left(I_{z} \sim 1 / \sqrt{\kappa x}\right.$ for $\kappa x \gg 1$; see Büchner and Zelenyi, 1990). Thus, we can combine the dependence of $\kappa x_{m}$ on $I_{z}$ and the dependence of $\kappa \bar{x}$ on $I_{z}$ to obtain $\tilde{I}_{z}$ as a solution of the equation $\kappa \bar{x}=\kappa x_{m}$ (Fig. 3, right panel). One can see that for each $\lambda$ we have two values of $\tilde{I}_{z}$. Furthermore we use the larger $\tilde{I}_{z}$.

All particles with $I_{z}<\tilde{I}_{z}$ penetrate into the CS from the boundaries. These particles approach the uncertainty curve, cross it, and accomplish the half-turn around $B_{z}$ field. Then the particles can be captured in the left potential well (in this case the particles reach the same coordinate $\kappa x_{m}$, but already with $z<0$ ) or can be captured in the right potential well (in this case the particles increase their invariant $I_{z}$ and the new value of $\kappa x_{m}$ becomes smaller). In the first case we deal with transition of the particles from one boundary of the CS $z=\lambda$ to another boundary $z=-\lambda$ (see the trajectory in Fig. 4a). In the second case the particles can become trapped in the CS (if the new value of $\kappa x_{m}$ is smaller than $\kappa \bar{x}$; see Fig. 4b) or can reach the same boundary $z=\lambda$ and be reflected from the CS (if the new value of $\kappa x_{m}$ is larger than $\kappa \bar{x}$, Fig. 4c).

A double crossing of the uncertainty curve (i.e. a double separatrix crossing) in the symmetric system with $s=0$ results only in a small variation $\sim \kappa$ of $I_{z}$. Thus, trajectories (transient and reflected) return to approximately the same coordinate $\kappa x_{m}$ : if particles come from the CS boundaries, then they return to these boundaries. Hence, particles can transit through the CS or can be reflected from it. In an asymmetric system with $s \neq 0$ there exist also geometrical jumps of $I_{z}$. As a result, after a double separatrix crossing, $I_{z}$ remains approximately the same for transient particles, which start in 
$s=0.1$
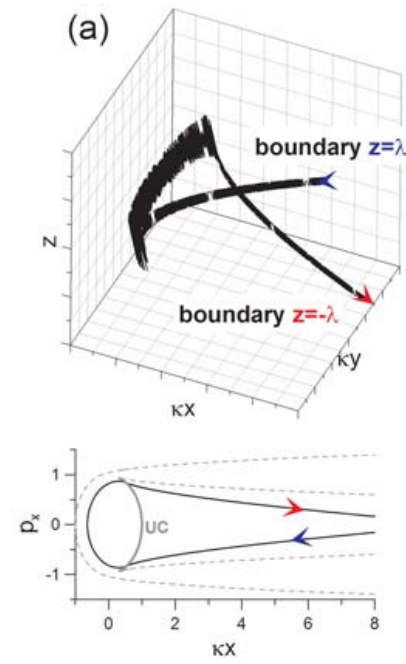

$s=0.1$

(b)
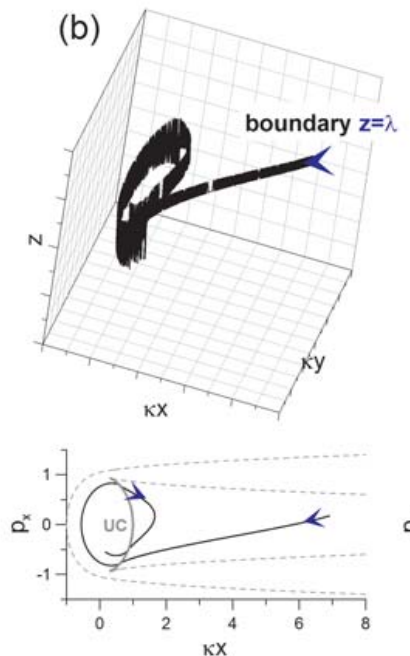

$s=0.05$
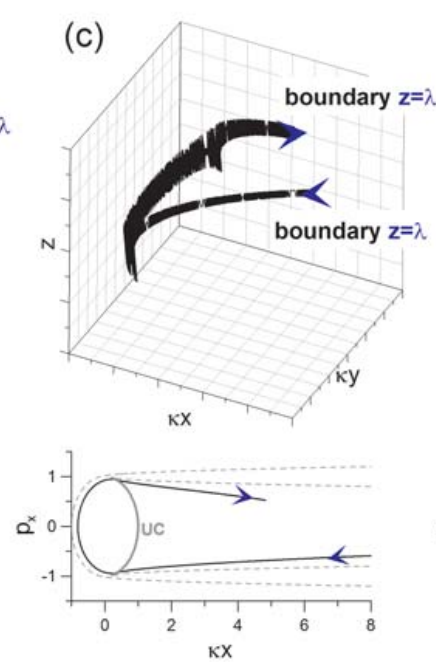

$\mathrm{s}=0.1$
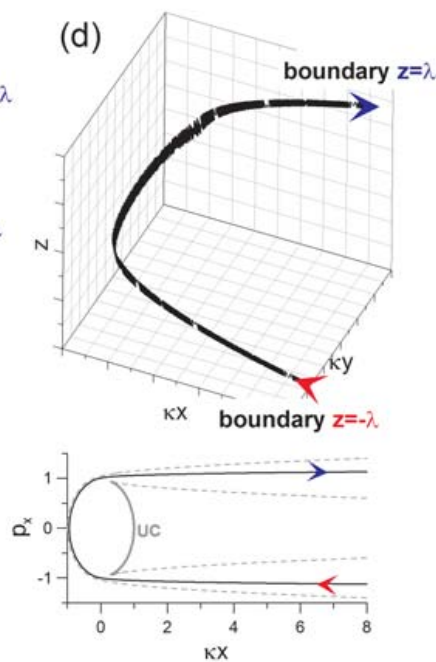

Fig. 4. Four types of trajectories and their projections onto the $\left(\kappa x, p_{x}\right)$ plane.

the right potential well $(z=\lambda)$ and are captured in the left potential well (i.e. reach the boundary $z=-\lambda$ ). For reflected particles (which become captured in the right potential well), the value of $I_{z}$ increases. Thus, such particles already cannot reach the initial coordinate $\kappa x_{m}$. Therefore, the particles starting from the boundary $z=\lambda$ are more likely to cross the $\mathrm{CS}$ than be reflected from it.

\subsection{The second scenario}

We consider now particles starting from the boundary $z=$ $-\lambda$. In this case the particles approach the neutral plane without crossing the uncertainty curve due to the shrinking of the uncertainty curve for $s \neq 0$ (see scheme in Fig. 5). Such particles do not accomplish a half-turn around $B_{z}$, but oscillate around field lines. An example of such a trajectory is shown in Fig. 4d. This type of motion resembles the classical gyrocentre motion without the demagnetization in the neutral plane. Therefore, particles starting at $z=-\lambda$ cannot be reflected from the CS and transit through it. This scenario is realized for realistic position of boundaries $(\lambda \geq 1)$ and not very small $B_{y}\left(s>\left(\pi^{-1} \ln 2\right) \kappa\right)$.

\subsection{The third scenario}

This scenario concerns particles trapped within the CS. For these particles the coordinates of mirror points $\kappa x_{m}$ are smaller than the corresponding coordinate of the boundary $\kappa \bar{x}$. There are two possible subscenarios of motion for such particles.

Due to geometrical jumps of $I_{z}$ the particles can change $I_{z}$ value at the uncertainty curve, and thus change the corresponding mirror points $\kappa x_{m}$. However, for each trajectory the number of such jumps with changing the value of $I_{z}$ is finite,

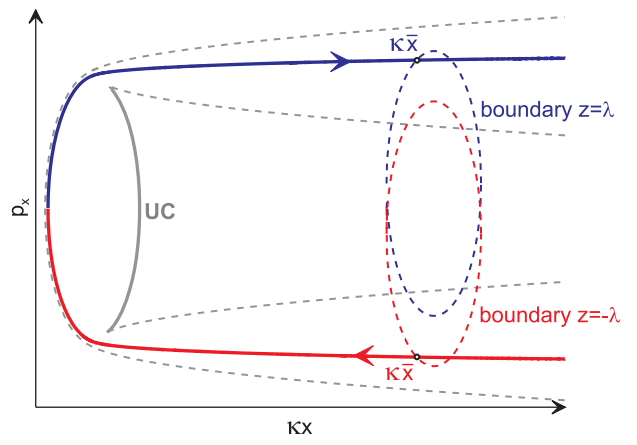

Fig. 5. Schematic view of particle trajectory.

i.e. the number of possible values of $I_{z}$ is finite. This number depends only on the $s$ parameter (see Artemyev et al., 2013). Therefore, there are particles with all possible values of $\kappa x_{m}$ smaller than $\kappa \bar{x}$ (see the scheme in Fig. 6a). These particles cannot escape from the CS, and only quasi-random dynamical jumps of $I_{z}$ may change this situation (see the next section). An example of such a trajectory is shown in Fig. 7a. In the absence of dynamical jumps of $I_{z}$, these particles would have been trapped inside the CS forever.

The second subscenario corresponds to particles with $I_{z}$ decreasing due to geometrical jumps so substantially that the new mirror points appear at $\kappa x_{m}>\kappa \bar{x}$ (see the scheme in Fig. 6b). These particles can make a half-turn around $B_{z}$ and escape from the CS through the boundary $z=-\lambda$ (an example of such trajectories is shown in Fig. 7b). 

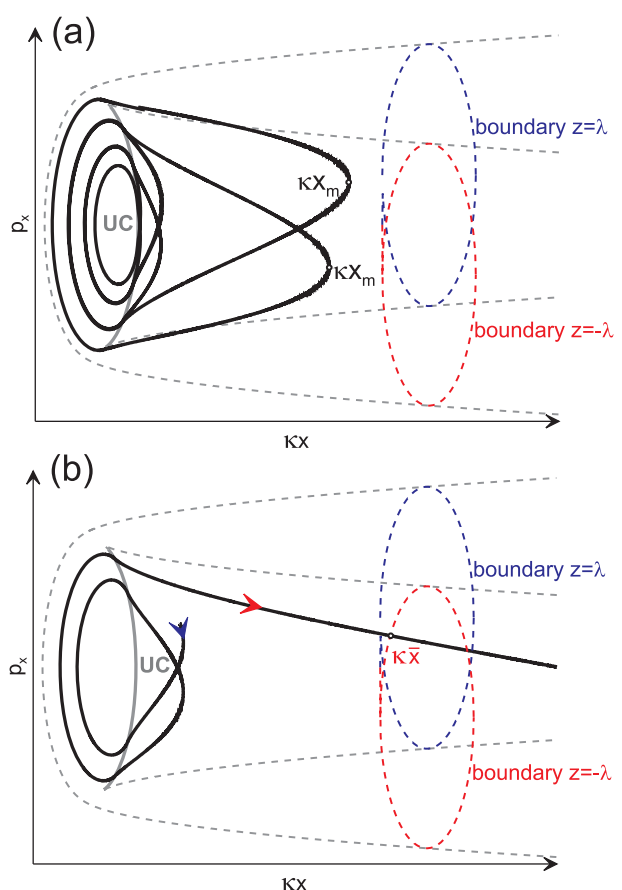

Fig. 6. Schematic view of particles' trajectories.
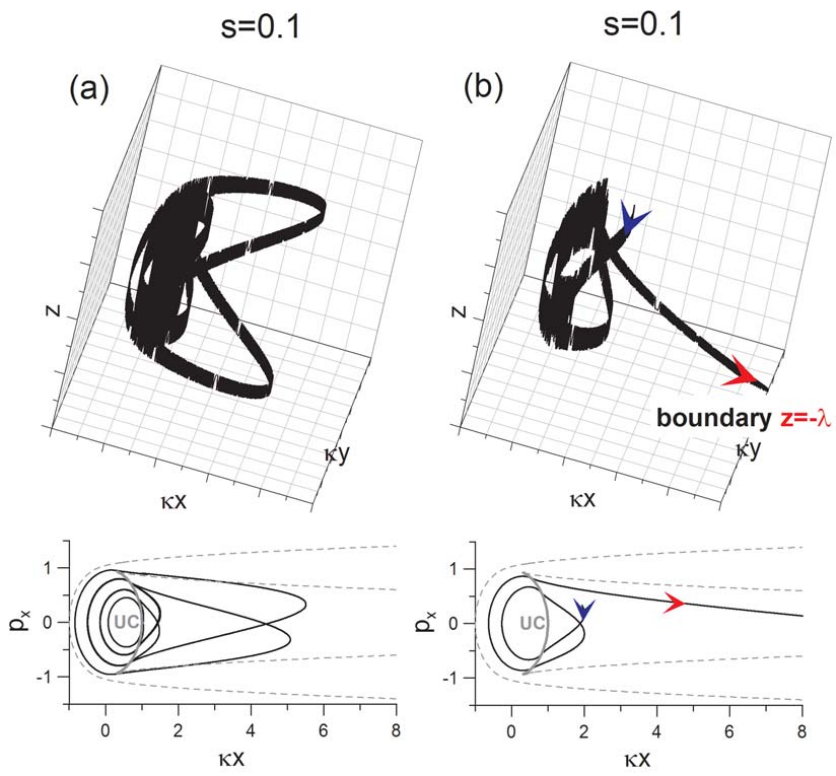

Fig. 7. Particles' trajectories and corresponding projections onto the $\left(\kappa x, p_{x}\right)$ plane.

\section{Destruction of the quasi-adiabatic invariant}

The quasi-adiabatic invariant $I_{z}$ is an approximate invariant of motion. Far from the separatrix, $I_{z}$ is conserved with the accuracy $\sim \kappa$ (see Arnold et al., 2006). One can introduce the improved quasi-adiabatic invariant $J=I_{z}+\kappa u$ where $u\left(z, p_{z}, \kappa x, p_{x}\right)$, is defined at each point of the phase space
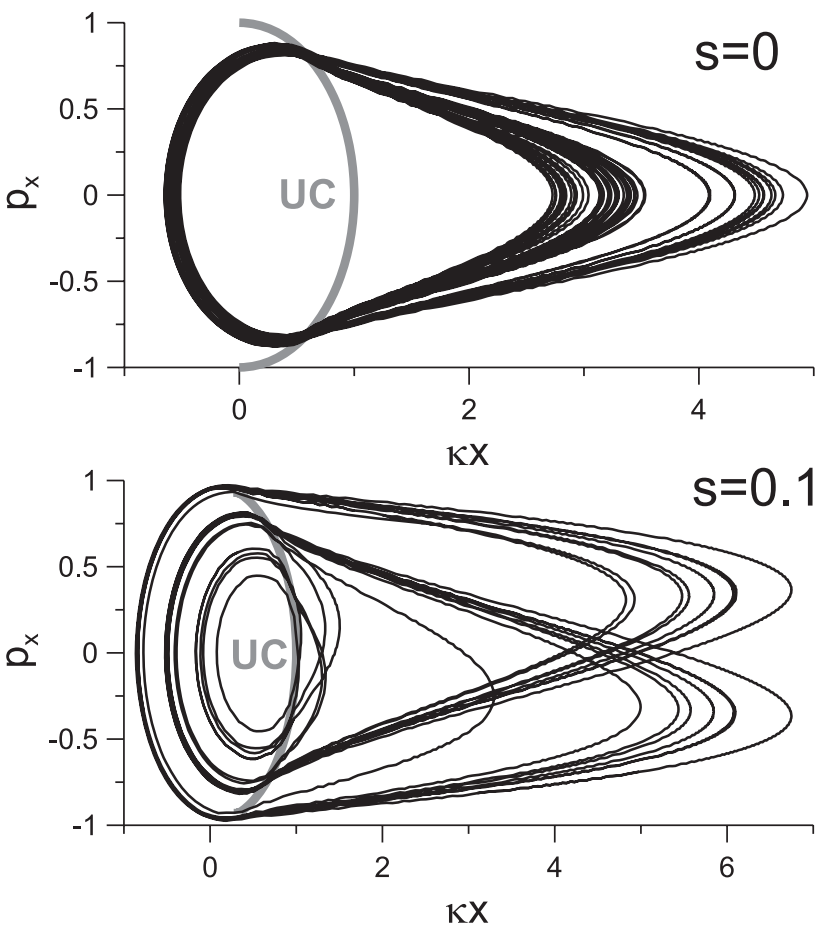

Fig. 8. Trajectories of two particles in the system with $\kappa=0.01$.

$\left(z, p_{z}, \kappa x, p_{x}\right)$ for fixed energy $H=1 / 2$ ( $u$ cannot be determined only at the separatrix). Far from the separatrix, $J$ is conserved with the accuracy $\sim \kappa^{2}$. Function $u$ is defined in Appendix A2.

As a result of separatrix crossings, the invariant $J$ experiences a jump $\Delta J=\Delta J^{\text {geom }}+\Delta J^{\text {dyn }}$, where the geometrical jump $\Delta J^{\text {geom }}$ is defined by the system geometry, and the dynamical jump $\Delta J^{\text {dyn }} \sim \kappa \ln \kappa$ depends on a variable $\xi \in(0,1)$, which characterizes the precise position of a separatrix crossing in the plane $\left(z, p_{z}\right)$, and can be considered as a quasi-random variable (see Appendix A). Thus, values of dynamical jumps can be treated as random. Dynamical jumps result in destruction of the quasi-adiabatic invariant, i.e. particles slightly change their trajectories in the plane $\left(\kappa x, p_{x}\right)$ at every crossing of the uncertainty curve. Examples of particle trajectories calculated on a long-time interval are shown in Fig. 8 for two values of $s$. One can see that both particle trajectories acquire the spread over the plane $\left(\kappa x, p_{x}\right)$. For a sufficiently long time interval the particle trajectory should fill a substantial part of the plane $\left(\kappa x, p_{x}\right)$ (the area of this covered part does not depend on $\kappa)$.

To demonstrate the effect of $\Delta J^{\text {dyn }}$ we calculate the particle trajectory on a long time period and show distributions of $I_{z}$ values measured at the moment of particle crossing of the neutral plane $z=0$ with $p_{x}=0$ (see Fig. 9). For the systems with $s \neq 0$ and a very small value of $\kappa$ we have several values of $I_{z}$. This is the effect of trajectory splitting due to the presence of geometrical jumps. There are also narrow 

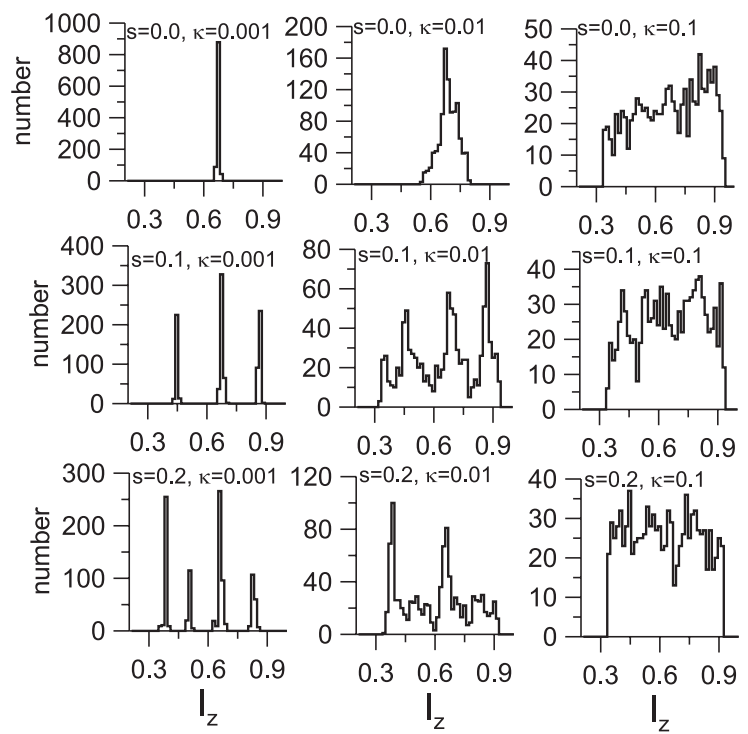

Fig. 9. Distributions of $I_{z}$ at various values of $s$ and $\kappa$. Each distribution contains $10^{3}$ values.

(but having a finite width) distributions around these maxima. This is the effect of $I_{z}$ destruction due to dynamical jumps. For the system with $s=0$ we observe the same result, but with the single peak value of $I_{z}$. An increase of $\kappa$ results in the increase of the width of $I_{z}$ distribution due to intensification of the destruction of $I_{z}$ because of dynamical jumps. The spreading of the $I_{z}$ distribution around the initial value (this value corresponds to the maximum of the distribution at $s=0$ ) is similar for systems with $s=0$ and $s \neq 0$. However, due to splitting of trajectories caused by geometrical jumps (the appearance of several maxima in the $I_{z}$ distribution for systems with $s \neq 0$ ), the whole range of accessible values of $I_{z}$ is wider for systems with $s \neq 0$. In the case of $\kappa=0.1$, dynamical jumps become comparable with geometrical jumps. As a result, there is a strong stochastization of particle motion.

\section{Statistical aspects of particle motion}

In this section we describe the probabilistic nature of particle captures in the potential wells at the uncertainty curve. When particles leave the neutral plane and cross the uncertainty curve, they can enter one of the two small potential wells. Capture in the left well corresponds to motion towards the bottom boundary $z=-\lambda$, while capture in the right well corresponds to motion towards the top boundary $z=\lambda$. For each trajectory this choice is determined by the coordinates of the separatrix crossing in the plane $\left(z, p_{z}\right)$. Because variables $\left(z, p_{z}\right)$ evolve fast and periodically, even a small variation of initial coordinates can result in a different choice of the potential well. Thus, the choice of the left or right potential wells can be considered as a probabilistic process
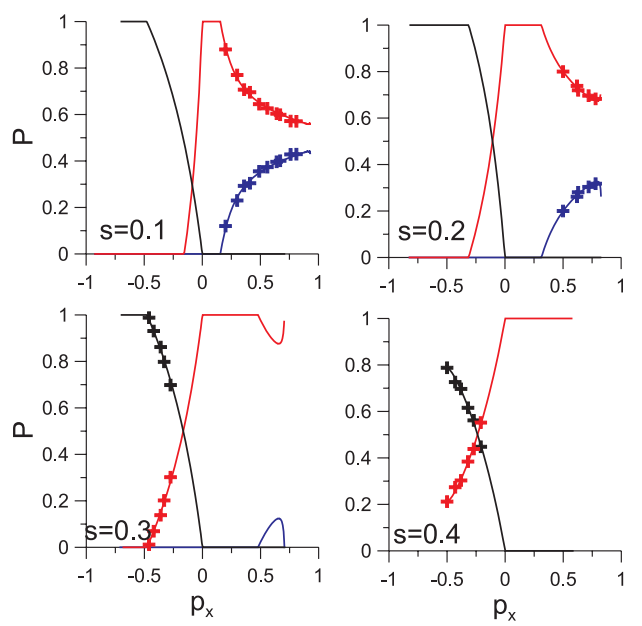

Fig. 10. Analytical profiles of probabilities as functions of $p_{x}$ along the uncertainty curve. Crosses show numerical results. Black colour is used for $P$, red for $P_{1}$, and blue for $P_{\mathrm{r}}$.

with certain probabilities $P_{1} \geq 0, P_{\mathrm{r}} \geq 0$. When particles approach the uncertainty curve being within one of the two possible potential wells, there is also a certain probability of capture in the single well $P=1-P_{\mathrm{r}}-P_{1}$. Analytical expressions for these probabilities were derived in Artemyev et al. (2013). Probabilities $P_{1, \mathrm{r}}, P$ depend on coordinates of the uncertainty curve crossing in the plane $\left(\kappa x, p_{x}\right)$. Probabilities $P_{1, \mathrm{r}}$ are positive if areas surrounded by corresponding separatrix loops (see Fig. 1) are growing. If areas decrease, then the corresponding probabilities are equal to zero.

For $s=0$ we have the symmetric system with $P_{1}=P_{\mathrm{r}}=$ 0.5 for $p_{x}>0$ and $P=1$ for $p_{x}<0$. For four values of $s>0$ we plot $P_{\mathrm{r}, 1}$ and $P$ as functions of $p_{x}$ along the uncertainty curve (see Fig. 10). With the increase of $s$ the probability $P_{\mathrm{r}}$ decreases, and for $s>0.35$ we have $P_{\mathrm{r}}=0$. Thus, for $s>$ 0.35 , particles cannot be captured in the right potential well at the uncertainty curve. Also for $s \neq 0$ we have $P_{1}>0$ for $p_{x}<0$. Thus, when particles approach the uncertainty curve while inside the right small well, they can be captured either in the single well or in the small left well.

To check the analytical expressions for the probabilities, we use two simulations with ensembles of particles. In the first simulation we take $s=0.1$ and $s=0.2$. We run $10^{4}$ particles with the same quasi-adiabatic invariant, the same energy, and the uniform distribution of initial coordinates along the trajectory in the plane $\left(z, p_{z}\right)$, i.e. a number of particles in a small trajectory fragment centred at a certain value of $p_{z}$ is inverse proportional to $p_{z}$ value. All the particles are initially located inside the single potential well $\left(p_{x}=0\right.$, $\kappa x<0)$. Then we collect these particles after crossings of the uncertainty curve and determine relative number of particles inside each of the two small potential wells. This simulation is repeated with various initial values of $\kappa x$ (i.e. with various values of the quasi-adiabatic invariant). Thus, we 
obtain numerical "effective" probabilities for several crossing points of the uncertainty curve (here we use the term "effective" to separate numerical data and analytical probabilities). These values are shown in Fig. 10 for $s=0.1, s=0.2$ by red and blue crosses. For the second simulation we use $s=0.3$ and $s=0.4$. We run $10^{4}$ particles inside the left potential well with the initial coordinates $p_{x}=0, \kappa x>1$. In this case particles approach the uncertainty curve and can be captured either in the single or left potential wells. We calculate corresponding effective probabilities (see black and red crosses in Fig. 10 for $s=0.3, s=0.4$ ). One can see that numerical results agree with analytical expressions quite well.

To illustrate the probabilistic nature of a choice of potential wells (where particles are captured), we present three realizations of one particle trajectory. All these realizations start from the same point in the plane $\left(\kappa x, p_{x}\right)$ (see Fig. 11). The black arrows show the start and finish points of trajectories. Red arrows indicate points where the particle changes the potential wells. Corresponding projections of particle trajectories in the plane $\left(\kappa x, p_{x}\right)$ are shown in Fig. 12.

Let us describe the trajectories in Figs. 11 and 12. First, the particle starts moving inside the right potential well. Then the particle approaches the uncertainty curve at $p_{x} \approx-0.4$. At this point the particle should leave the right well, because the corresponding area decreases (see detailed description of area and probability distributions along the uncertainty curve in Artemyev et al., 2013). Areas of the left well and the single well are increasing, and thus there are certain possibilities to be captured in the single well with the probability $P$ and in the left well with the probability $P_{l, 1}$. In the first case the particle accomplishes a half-turn around $B_{z}$ and approaches the uncertainty curve while within the single well with $p_{x} \approx$ 0.4. At this point the particle can be captured only in the left well (as the area of the right well decreases). This is the realization (A) in Fig. 11.

In the second case the particle is reflected from the uncertainty curve inside the left well with $p_{x} \approx-0.4$, and approaches this curve again with $p_{x} \approx-0.6$. At this point the areas of both small potential wells decrease and the particle can be captured only in the single well. Then the particle makes a half-turn around $B_{z}$ and approaches the uncertainty curve inside the single potential well with $p_{x} \approx 0.6$. At this point the areas of both small potential wells increase and the particle can be captured in the right well with the probability $P_{r, 2}$ and in the left well with the probability $P_{l, 2}$. In the first case we have the realization (B), and the second case corresponds to the realization (C) in Fig. 11. Such splittings of the single trajectory into three realizations are possible only for the non-symmetric system with $s \neq 0$ when the two areas of small potential wells evolve asynchronously.

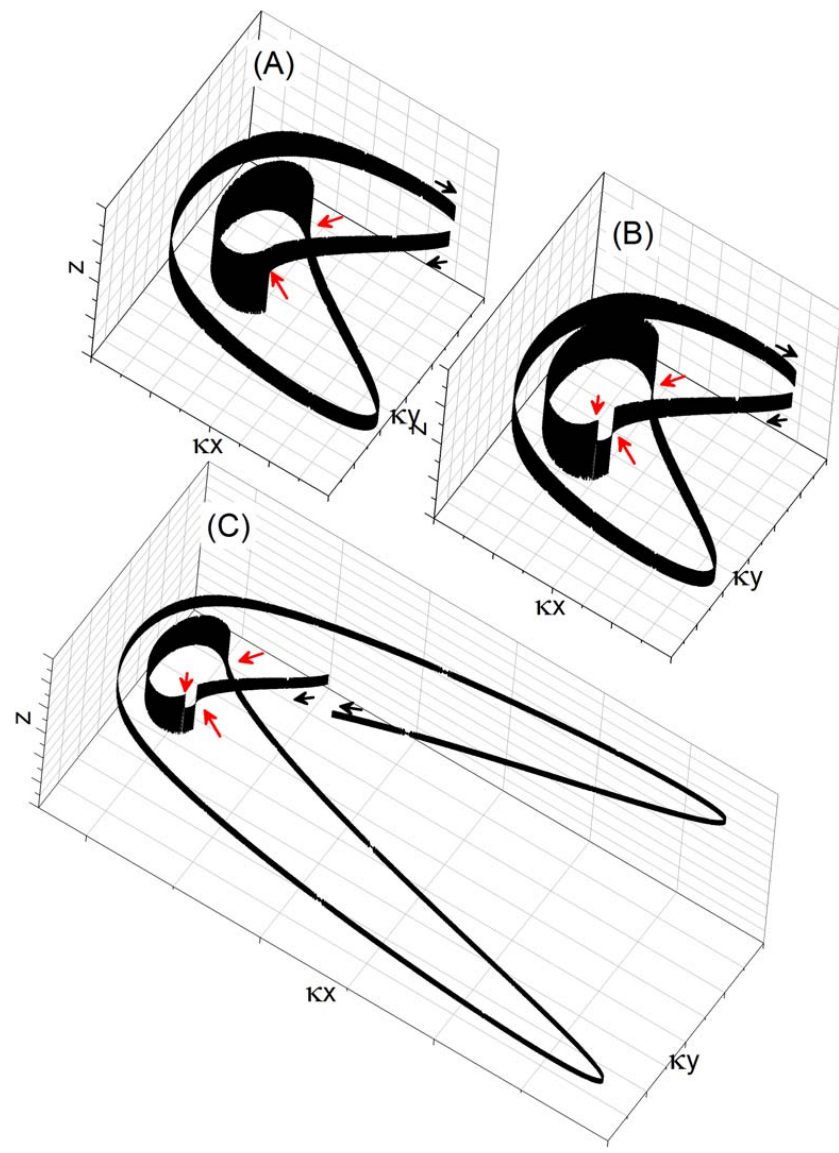

Fig. 11. Three realizations of particle trajectories starting from one point in the plane $\left(\kappa x, p_{x}\right)$.

\section{Discussion}

Trapped and transient particles play different roles in the CS. Trapped particles with reflection points below the CS boundaries $|z|=\lambda$ accomplish their oscillation motion inside the CS (their adiabatic trajectories with $I_{z}=$ const are closed in the $\left(\kappa x, p_{x}\right)$ plane). As a result, the total electric current carried by these particles is exactly equal to zero (Pellat and Schmidt, 1979). However, local currents of trapped particles are not equal to zero. These currents are positive at some distance from the neutral plane and negative in the vicinity of the neutral plane (Zelenyi et al., 2000). In contrast, orbits of transient particles crossing the boundaries $|z|=\lambda$ are open. Thus, the total current of these particles is not equal to zero and has a strong maximum in the vicinity of the neutral plane (Eastwood, 1972). As a result, the self-consistent CS equilibrium can exist only if the population of transient particles is large enough in comparison with the population of trapped particles (see discussion in Burkhart et al., 1992; Zelenyi et al., 2002b, c). Transient particles can be scattered and trapped within the CS due to random dynamical jumps of $I_{z}$. Therefore, in the absence of any mechanism of 

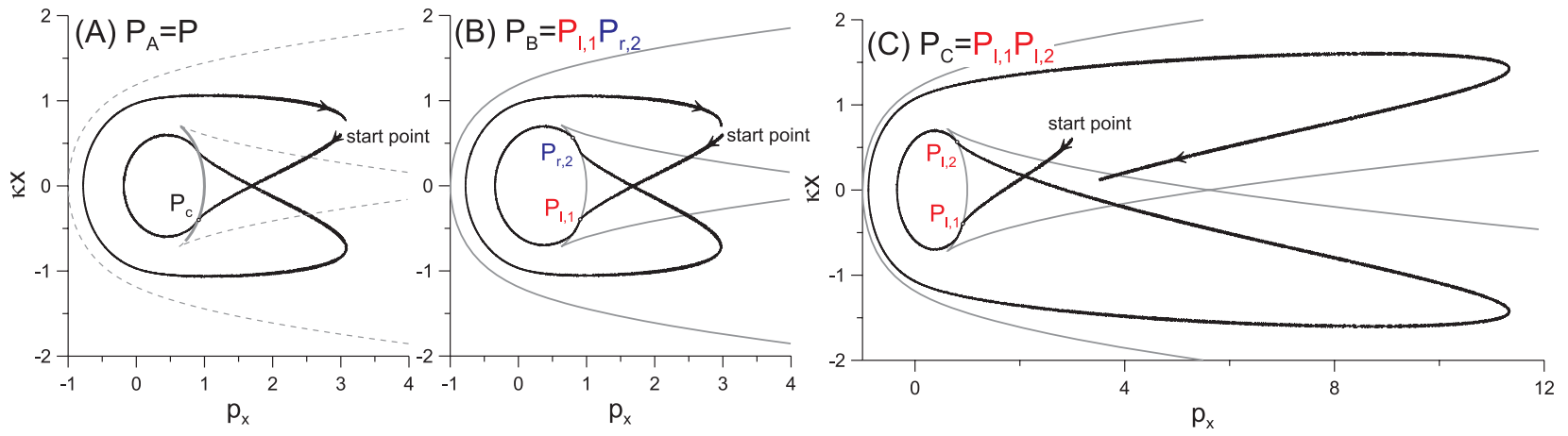

Fig. 12. Three panels show projections of trajectories from Fig. 11 to the plane $\left(\kappa x, p_{x}\right)$. Probabilities of all trajectories corresponds to combination of probabilities at the uncertainty curve.

particle detrapping, the CS lifetime is limited by the time of total stochastization of particle motion (Zelenyi et al., 2002a, 2003). We obtain an important result for dynamical jumps in the CS with $B_{y} \neq 0$ : in contrast to the symmetric system, where $\left\langle\Delta J^{\text {dyn }}\right\rangle=0$, in asymmetric systems we have $\left\langle\Delta J^{\text {dyn }}\right\rangle \neq 0$ (see Appendix A). This effect drastically changes the characteristic time of stochastization of particle motion. $I_{z}$ of each particle changes by a value $\sim \kappa$ (here for simplicity we omit $\ln \kappa$ ) in the course of one crossing of the uncertainty curve (one crossing of the separatrix). During one period of motion in the $\left(\kappa x, p_{x}\right)$ plane (the period is $\sim \kappa^{-1}$ ) particles are crossing the uncertainty curve twice. If $\left\langle\Delta J^{\text {dyn }}\right\rangle=0$ ( $s=0$ case), then the averaged jump is equal to zero, but $\left\langle\left(\Delta J^{\mathrm{dyn}}\right)^{2}\right\rangle \sim \kappa^{2}$ is finite. Thus, we need time $\sim \kappa^{-3}$ to change the invariant value substantially (the situation is different in the special case of when an initial value of $I_{z}$ is comparable with $\kappa$; see Vainshtein et al., 1999; Vainchtein et al., 2005). In the case of non-zero average value $\left\langle\Delta J^{\mathrm{dyn}}\right\rangle \neq 0(s \neq 0$ case $)$ there is a drift in the space of the invariants. This drift results in effective evolution of $I_{z}$, and as a result, the time required for the substantial change of $I_{z}$ is about $\kappa^{-2}$. For parameters of the Earth's magnetotail this effect results in the decrease of the stochastization time for one order of magnitude. Previous estimates gave the time of the CS destruction due to stochastization around tens of minutes for $\kappa \leq 0.1$ (Zelenyi et al., 2002a, 2003). In the case of finite $B_{y}$ (for $B_{y} \geq B_{z}$ ) the time of stochastization of particle motion (and corresponding CS destruction) becomes of the order of a few minutes. This is a rather small time interval for the Earth's magnetotail. Thus, the existence of the CS with small but finite $B_{y}\left(\left(\pi^{-1} \ln 2\right) \kappa<s<0.35\right)$ seems to be impossible without a certain mechanism of particle detrapping. The role of this mechanism can be played by the earthward convection, when trapped particles get a chance to escape from the CS region due to the earthward drift motion. In addition, any (even weak) gradient of $B_{z}$ along the $x$ axis results in the drift of trapped particles in the $y$ direction. In this case trapped particles can already contribute to the total cross-tail current, and thus help support the CS configuration (see discussion in Artemyev and Zelenyi, 2013).

Increase of the stochastization rate of particle motion with the increase of $B_{y}$ (until $s<0.35$ ) is also important due to the additional role played by the trapped population. The transverse electric field exists in the Earth's magnetotail (see Kan, 1990; Angelopoulos et al., 1993) and in reconnected CSs of the solar corona (e.g. Litvinenko, 1996). Thus, stochastic motion of trapped particles can contribute to the transverse collisionless conductivity in the CS (Horton and Tajima, 1990). The magnitude of such conductivity strongly depends on the level of stochasticity of particle motion (Holland and Chen, 1992; Greco et al., 2000; Numata and Yoshida, 2002). Thus, enhancement of stochastization should result in an increase of collisionless conductivity and support the development of various resistive instabilities in the CS (see review by Horton, 1997, and references therein).

Although we obtain non-zero $\left\langle\Delta J^{\text {dyn }}\right\rangle$ (averaging over $\xi$; see Appendix A), we should also take into account the conservation of phase volume in the system. This requirement can be written as a kinetic equation $\mathrm{d} f / \mathrm{d} t=0$ for the distribution function of particles $f$, where $d / \mathrm{d} t$ is the total derivative (Pitaevskii and Lifshitz, 1981). Absence of particle collisions results in the conservation of the phase volume, and forbids directed drifts of particles in the invariant space (Sinitsyn et al., 2011), i.e. the double averaged $\left\langle\left\langle\Delta J^{\mathrm{dyn}}\right\rangle\right\rangle_{I_{z}}$ (the second averaging is performed over adiabatic invariants) should be equal to zero. Therefore, we obtain that for each $I_{z}$ an averaged value $\left\langle\Delta J^{\mathrm{dyn}}\right\rangle$ is non-zero, but for all population of particles we have only redistribution of invariants without the appearance of any particle fluxes in the phase space. However, we should mention that the presence of the boundaries $z= \pm \lambda$ can result in non-zeroaveraged jumps $\left\langle\left\langle\Delta J^{\mathrm{dyn}}\right\rangle\right\rangle_{I_{z}} \neq 0$ with corresponding particle drift in the phase space (Zelenyi et al., 2003).

The effect of asymmetry of particle reflection/transition in the CS with $B_{y} \neq 0$ has been mentioned by many authors on the basis of numerical simulations (Zhu and Parks, 1993; Baek et al., 1995; Delcourt et al., 2000; Malova et al., 2012; 
Grigorenko et al., 2013). Here we have shown that this effect occurs due to two peculiarities of the system: (1) decrease of the probability of capture in the right well and (2) shrinking of the uncertainty curve. For particles coming to the CS with $B_{y}>0$ from the Northern Hemisphere (from the boundary $z=\lambda$ ) the probability to transit through the CS is larger for larger $B_{y}$. For $s>0.35$ (i.e. $B_{y}>0.35 B_{0} \sqrt{L / \rho_{0}}$ ) particles from the Northern Hemisphere cannot be reflected from the $\mathrm{CS}$ at all. These particles either should cross the sheet and come to the boundary $z=-\lambda$ or should be trapped in the CS with decrease of the distance between the neutral plane and positions of corresponding reflection points. A decrease in the length of the uncertainty curve with the increase of $B_{y}$ results in the absence of the uncertainty curve crossings for particles from the Southern Hemisphere (from the boundary $z=-\lambda$ ). These particles cannot be reflected from the CS and even cannot be scattered in the CS. Their trajectories cross the neutral plane without a half-rotation around $B_{z}$. Thus, such particles come directly to the boundary $z=\lambda$ (normally gyrating around field lines). Roughly speaking, in systems with $B_{y} \neq 0$, the probability of particle reflection from the CS to the initial hemisphere decreases with the increase of $B_{y}$. For large enough $B_{y}>B_{0} \sqrt{L / \rho_{0}}$, the scattering of particles is absent (see Artemyev et al., 2013). Thus, all particles cross the neutral plane moving along trajectories, which can be described by the guiding-centre theory. This effect of asymmetry of the CS interaction with particles can play an important role in the Earth's magnetotail, where $B_{y}$ is provided by penetration of the interplanetary magnetic field (Cowley, 1981; Wing et al., 1995), by deformation of the neutral plane (Petrukovich, 2009) or by local currents (Artemyev, 2011; Rong et al., 2012). Particles usually come to the CS of the Earth's magnetotail from the sources in the Southern and Northern Hemisphere. If these sources have different intensities, but $B_{y}=0$, then symmetric reflection/transition results in symmetric field-aligned flows of particles in both hemispheres. However, even small $B_{y} \neq 0$ results in asymmetric reflection and asymmetric flows of ions from the magnetotail towards the ionosphere. Auroral phenomena in the ionosphere are often considered as projections of particle flows from the magnetotail (see, e.g. Østgaard and Laundal, 2012, and references therein). Thus, the asymmetry of auroral phenomena in the case of $B_{y} \neq 0$ (Liou and Newell, 2010; Lukianova et al., 2012) can be partially explained by asymmetry of CS interaction with ions and corresponding asymmetry of compensation of electron currents.

One of the most beautiful manifestations of the nonlinear particle dynamics in the CS is the so-called resonant interaction: particles with a certain value of energy (i.e. the certain value of $\kappa$ ) are not scattered in the CS (Chen and Palmadesso, 1986; Burkhart and Chen, 1991; Büchner, 1991). This resonant effect is responsible for the formation of beamlets - coherent beams of accelerated particles (Ashour-Abdalla et al., 1992; Grigorenko et al., 2005, 2011). Resonant interaction can be explained by a compensation of two successive dynamical jumps of the quasi-adiabatic invariant (e.g. Büchner and Zelenyi, 1989; Zelenyi et al., 2007). The theory of such resonances was derived for the symmetric CS with $s=0$ (e.g. Ashour-Abdalla et al., 1993; Dolgonosov et al., 2010). For systems with $B_{y} \neq 0\left(s>\left(\pi^{-1} \ln 2\right) \kappa\right)$, various numerical calculations have shown that resonances could be destroyed (e.g. Kaufmann et al., 1994; Holland et al., 1996). We describe this destruction using the modification of the theory of compensation of successive dynamical jumps for systems with $s>\left(\pi^{-1} \ln 2\right) \kappa$ (see Appendix B). It can be shown that for $s>\left(\pi^{-1} \ln 2\right) \kappa$ and fixed $\kappa$, the simultaneous compensation of two successive dynamical jumps is possible only for relatively small group of particles with a certain value of the quasi-adiabatic invariant. The population of resonant particles for fixed $\kappa$ decreases with the increase of $s$. As a result, the effect of resonant interaction with the CS cannot be seen for a large population of particles in systems with $s>\left(\pi^{-1} \ln 2\right) \kappa$.

\section{Conclusions}

In this paper we have described effects of the magnetic field shear on non-adiabatic behaviour of charged particles. The main conclusions are listed below:

1. The presence of $B_{y} \neq 0$ results in asymmetry of particle reflection from (and transition through) the CS. For $B_{y}>0$, particles from the Southern Hemisphere $(z>0)$ are more likely to cross the CS. The probability of the CS crossing for these particle increases with the growth of $B_{y}$ and for $B_{y}>0.35 B_{0} \sqrt{L / \rho_{0}}$ all particles from the Southern Hemisphere are crossing the CS. Particles from the Northern Hemisphere are crossing the CS without scattering already for $s>\left(\pi^{-1} \ln 2\right) \kappa$ (i.e. $\left.B_{y}>\left(\pi^{-1} \ln 2\right) B_{z}\right)$. These particles cannot be reflected from the CS in the case of $B_{y}>0$. The situation is mirror symmetric for $B_{y}<0$ (Southern Hemisphere $\longleftrightarrow$ Northern Hemisphere).

2. In systems with $B_{y} \neq 0$ the intensification of particle scattering (and corresponding chaotization of motion) occurs. Average values of dynamical jumps of the quasi-adiabatic invariant are not equal to zero. The presence of geometrical jumps due to the emerging effective asymmetry of the system with $B_{y} \neq 0$ helps to destroy the adiabaticity.

3. Finite $B_{y}>\left(\pi^{-1} \ln 2\right) B_{z}$ destroys the resonances in the CS. In systems with large enough $B_{y}$ the resonant condition (compensation of two successive dynamical jumps of the quasi-adiabatic invariant) cannot be satisfied simultaneously for a large population of particles.

All these results are valid for systems with $s<1$. For $s>1$ the uncertainty curve (and the separatrix) disappears and the motion of charged particles is regular. 


\section{Appendix A}

\section{Jumps of the quasi-adiabatic invariant}

In this appendix we derive expressions for jumps of the quasi-adiabatic invariant. These expressions depend on areas $S_{\mathrm{l}, \mathrm{r}}$ of the separatrix loops and rates of their evolution $\kappa \Theta_{\mathrm{r}, 1}$ (see scheme in Fig. A4). Areas $S_{1, \mathrm{r}}$ and rates $\kappa \Theta_{\mathrm{r}, 1}$ are functions of slow variables $\left(\kappa x, p_{x}\right)$. However, below we define $S_{\mathrm{l}, \mathrm{r}}, \kappa \Theta_{\mathrm{r}, 1}$ only along the uncertainty curve. Thus, $S_{\mathrm{l}, \mathrm{r}}, \kappa \Theta_{\mathrm{r}, 1}$ depend on the $p_{x}$ coordinate along the uncertainty curve. Expressions for $S_{1, \mathrm{r}}$ and $\Theta_{\mathrm{l}, \mathrm{r}}$ were derived in Artemyev et al. (2013):

$$
\begin{aligned}
& S_{1, \mathrm{r}}=-A_{s} \pm 2 \pi z_{c}\left(g_{c}^{2}+z_{c}^{2}\right) \\
& A_{s}=-4 z_{c}\left(g_{c}^{2}+z_{c}^{2}\right) \arctan \left(\frac{z_{c}}{g_{c}}\right)-4 z_{c}^{2} g_{c}-\frac{8}{3} g_{c}^{3} \\
& \Theta_{1, \mathrm{r}}=2 A_{z_{c}}\left(2 A_{\Theta} \pm \pi s\right) \\
& A_{\Theta}=s \arctan \left(\frac{z_{c}}{g_{c}}\right)-\frac{z_{c}}{s} g_{c},
\end{aligned}
$$

where $g_{c}=g\left(z_{c}\right)$ and

$g(z)=\sqrt{A_{z_{c}}-s^{2}-\frac{1}{4}\left(z+z_{c}\right)^{2}}, A_{z_{c}}=\left(1+\frac{z_{c}^{2}}{s}\right)^{-1 / 2}$

We also introduce the asymptotic expression for the period of particle fast oscillations $T_{1, \mathrm{r}}=b_{\mathrm{l}, \mathrm{r}}-a \ln |E|$, where $E=$ $H-h_{c}$ and $h_{c}$ is the energy at the saddle point $z_{c}$ of the separatrix (see Fig. A4):

$h_{c}=\frac{1}{2}\left(p_{x}-s z_{c}\right)^{2}+\frac{1}{2}\left(\kappa x-\frac{1}{2} z_{c}^{2}\right)^{2}$.

Coefficients $b_{1, \mathrm{r}}$ and $a$ are derived in Sect. A1.

To evaluate jumps of the quasi-adiabatic invariant we have to introduce the improved invariant $J=I_{z}+\kappa u$, where the expression for $u$ is derived in Sect. A2. Finally, we use the relation between the value $J^{-}$of the improved invariant before crossing of the uncertainty curve and its value $J^{+}$after crossing. This relation can take several forms corresponding to different transitions. Here we write the general expression for relation between $J^{-}$and $J^{+}$(see Neishtadt, 1987) and then reduce it to the form corresponding to the Hamiltonian system (1).

For the transition from the single well to one of two small wells, when areas of both small wells grow, we have $2 \pi J^{-}=\left(S_{1}+S_{\mathrm{r}}\right)+\kappa u, J^{+}=J_{1, \mathrm{r}}$. Here areas $S_{1, \mathrm{r}}$ are defined at points where adiabatic trajectories (corresponding to the initial value of the invariant $I_{z}$ far from the uncertainty curve) cross the uncertainty curve. The relation between $J^{-}$and $J^{+}$ can be written as (Neishtadt, 1987)

$$
\begin{aligned}
& 2 \pi J_{1, \mathrm{r}}=S_{1, \mathrm{r}}+2 \pi \theta_{1, \mathrm{r}} \kappa \tilde{u}+\kappa\left(d_{1, \mathrm{r}}-\theta_{\mathrm{l}, \mathrm{r}}\left(d_{\mathrm{r}}+d_{1}\right)\right) \\
& +\kappa a \Theta_{1, \mathrm{r}}\left(\xi-\frac{1}{2}\right)\left(\ln \left(\kappa \Theta_{\mathrm{l}, \mathrm{r}}\right)-2 \theta_{1, \mathrm{r}} \ln (\kappa \Theta)\right) \\
& +\kappa a \Theta_{1, \mathrm{r}} \ln \left(\Gamma(\xi) \Gamma\left(\theta_{\mathrm{l}, \mathrm{r}}(1-\xi)\right) \Gamma\left(1-\theta_{\mathrm{l}, \mathrm{r}} \xi\right) /(2 \pi)^{3 / 2}\right) \\
& -\kappa \Theta_{1, \mathrm{r}}\left(\xi-\frac{1}{2}\right)\left(b_{1, \mathrm{r}}-\theta_{1, \mathrm{r}}\left(b_{\mathrm{r}}+b_{1}\right)\right) \\
& -\kappa \theta_{1, \mathrm{r}}\left(\xi-\frac{1}{2}\right)\left\{S_{1, \mathrm{r}}, S\right\}+O\left(\kappa^{3 / 2} \ln \kappa\right),
\end{aligned}
$$

where $d_{1, \mathrm{r}}=u_{1, \mathrm{r}} / 2 \pi=0$ (see Sect. A2), $\Theta=\Theta_{1}+\Theta_{\mathrm{r}}, \theta_{1, \mathrm{r}}=$ $\Theta_{1, \mathrm{r}} / \Theta,\{\ldots\}$ is the Poisson bracket, $\xi \in(0,1)$ is a uniformly distributed random value (see details in Arnold et al., 2006) and $\tilde{u}=J^{-}-S / 2 \pi \neq 0 . \Gamma$ is the gamma function. The transition from small wells to the single well in the case of $\Theta_{\mathrm{r}}<0, \Theta_{1}<0$ is described by the same Eq. (A1), where $J^{+}=J_{1, \mathrm{r}}$ and $S_{1, \mathrm{r}}=2 \pi J^{-}-\kappa u-S_{\mathrm{r}, 1}$.

Equation (A1) can be used to describe the transition from the single well to the left small well when the area of the right small well decreases (i.e. $\Theta_{\mathrm{r}}<0$ ). In this case we have $\Theta_{1}>0, \Theta>0$ and $\xi \in\left(0,1-\left|\Theta_{\mathrm{r}} / \Theta_{1}\right|\right)$. Equation (A1) also describes the transitions from the left small well to the single well (with the same relations $2 \pi J^{-}=\left(S_{1}+S_{\mathrm{r}}\right)+\kappa u, J^{+}=$ $J_{1, \mathrm{r}}$ ) when the area of the right well increases. In this case we have $\Theta_{\mathrm{r}}>0, \Theta_{1}<0, \Theta<0$ and $\xi \in\left(0,1-\left|\Theta_{\mathrm{l}} / \Theta_{\mathrm{r}}\right|\right)$.

Here we should mention that Eq. (A1) also contains a term $O\left(\kappa^{3 / 2}(1-\xi)^{-1}\right)$ (see Neishtadt, 1987). Below, we omit this term and assume that $\xi$ is far from 1 (i.e. $1-\xi \gg \kappa$ ).

For the transition between small wells (when $\Theta_{\mathrm{r}}<0$ and $\Theta_{1}>0$ ) we have (see Neishtadt, 1987)

$$
\begin{aligned}
& 2 \pi J_{1}=S_{1}+2 \pi \tilde{\theta}^{-1} \kappa \tilde{u}_{\mathrm{r}}+\kappa\left(d_{1}-\tilde{\theta} d_{\mathrm{r}}\right) \\
& +\kappa a(1-\xi)\left(\Theta_{\mathrm{r}} \ln \left(\kappa \Theta_{1}\right)-\Theta_{1} \ln \left|\kappa \Theta_{\mathrm{r}}\right|\right) \\
& -\kappa a \Theta_{1}(\ln (2 \pi(1-\xi) \sqrt{|\tilde{\theta}|})-\ln (\Gamma(\xi) \Gamma(1+\tilde{\theta}-\tilde{\theta} \xi))) \\
& +\kappa(1-\xi)\left(\Theta_{1} b_{\mathrm{r}}-\Theta_{\mathrm{r}} b_{1}\right) \\
& -\kappa(1-\xi)\left\{S_{1}, S_{\mathrm{r}}\right\}+O\left(\kappa^{3 / 2} \ln \kappa\right),
\end{aligned}
$$

where $d_{1, \mathrm{r}}=u_{1, \mathrm{r}} / 2 \pi=0$ (see Sect. A2), $\tilde{u}_{\mathrm{r}}=J_{\mathrm{r}}-S_{\mathrm{r}} / 2 \pi \neq 0$ and $\tilde{\theta}=\Theta_{\mathrm{r}} / \Theta_{\mathrm{l}}<0$. Here $\xi \in(0,1)$ for $\Theta>0$ and $\xi \in(1-$ $\left.\left|\Theta_{1} / \Theta_{\mathrm{r}}\right|, 1\right)$ for $\Theta<0$.

Terms $\left\{S_{1, \mathrm{r}}, S\right\}=\left\{S_{1}, S_{\mathrm{r}}\right\}$ and $\left\{S_{\mathrm{l}}, S_{\mathrm{r}}\right\}$ in Eqs. (A1) and (A2) are defined in Sect. A3. In Sect. A1 we show that $b_{1}$ is equal to $b_{\mathrm{r}}$ (we introduce $b=b_{1, \mathrm{r}}$ ). Then we have $b_{1, \mathrm{r}}-\theta_{1, \mathrm{r}}\left(b_{1}+\right.$ $\left.b_{\mathrm{r}}\right)=b\left(1-2 \theta_{\mathrm{l}, \mathrm{r}}\right)$. Finally, for the transition between the single well and small wells we have

$$
\begin{aligned}
\Delta J & =J_{1, \mathrm{r}}^{+}-J^{-}=\Delta J_{1, \mathrm{r}}^{\mathrm{geom}}+\Delta J_{1, \mathrm{r}}^{\mathrm{dyn}}+O\left(\kappa^{3 / 2} \ln \kappa\right) \\
\Delta J_{1, \mathrm{r}}^{\mathrm{geom}} & =\frac{1}{2 \pi}\left(S_{1, \mathrm{r}}-S\right)=-\frac{1}{2 \pi} S_{\mathrm{r}, \mathrm{l}} \\
\Delta J_{1, \mathrm{r}}^{\mathrm{dyn}} & =\kappa a \Theta_{1, \mathrm{r}}\left(\xi-\frac{1}{2}\right)\left(\ln \left(\kappa \Theta_{1, \mathrm{r}}\right)-2 \theta_{1, \mathrm{r}} \ln (\kappa \Theta)\right) \\
& -\kappa a \Theta_{1, \mathrm{r}} \ln \frac{(2 \pi)^{3 / 2}}{\Gamma(\xi) \Gamma\left(\theta_{1, \mathrm{r}}(1-\xi)\right) \Gamma\left(1-\theta_{1, \mathrm{r}} \xi\right)} \\
& -\kappa \Theta_{1, \mathrm{r}} b\left(\xi-\frac{1}{2}\right)\left(1-2 \theta_{1, \mathrm{r}}\right)+\kappa \theta_{1, \mathrm{r}} \tilde{u} \\
& -\kappa \theta_{1, \mathrm{r}}\left(\xi-\frac{1}{2}\right)\left\{S_{1, \mathrm{r}}, S\right\},
\end{aligned}
$$

and for the transition between small wells 


$$
\begin{aligned}
\Delta J & =J_{1}^{+}-J_{\mathrm{r}}^{-}=\Delta J^{\text {geom }}+\Delta J^{\mathrm{dyn}}+O\left(\kappa^{3 / 2} \ln \kappa\right) \\
\Delta J^{\text {geom }} & =\frac{1}{2 \pi}\left(S_{1}-S_{\mathrm{r}}\right) \\
\Delta J^{\mathrm{dyn}} & =\kappa a(1-\xi)\left(\Theta_{\mathrm{r}} \ln \left|\kappa \Theta_{\mathrm{l}}\right|-\Theta_{1} \ln \left|\kappa \Theta_{\mathrm{r}}\right|\right) \\
& -\kappa a \Theta_{1} \ln \frac{2 \pi(1-\xi) \sqrt{|\tilde{\theta}|}}{\Gamma(\xi) \Gamma(1+\tilde{\theta}-\tilde{\theta} \xi)} \\
& +\kappa(1-\xi) b\left(\Theta_{1}-\Theta_{\mathrm{r}}\right)+\tilde{\theta}^{-1} \kappa \tilde{u}_{\mathrm{r}} \\
& -\kappa(1-\xi)\left\{S_{1}, S_{\mathrm{r}}\right\} .
\end{aligned}
$$

Here we define the geometrical jump in Eqs. (A3) and (A4) as the difference of unperturbed areas surrounded by the separatrix loops before and after the separatrix crossing.

For the system with the symmetric phase portrait $(s=0)$ we have $S_{1}=S_{\mathrm{r}}=S / 2, \Theta_{1}=\Theta_{\mathrm{r}}=\Theta / 2$. Then the transition between two small wells is impossible. For the transition between the single well and one small well we have

$$
\begin{aligned}
\Delta J_{1, \mathrm{r}}^{\text {geom }} & =-\frac{1}{4 \pi} S=-\frac{1}{2} J^{-} \\
\Delta J_{1, \mathrm{r}}^{\mathrm{dyn}} & =-\frac{1}{2 \pi} \kappa a \Theta\left(\xi-\frac{1}{2}\right) \ln 2 \\
& -\frac{1}{2 \pi} \kappa a \Theta_{1, \mathrm{r}} \ln \frac{(2 \pi)^{3 / 2}}{\Gamma(\xi) \Gamma\left(\frac{1}{2}(1-\xi)\right) \Gamma\left(1-\frac{1}{2} \xi\right)} \\
& =-\frac{1}{2 \pi} a \kappa \Theta_{1, \mathrm{r}}\left(\xi-\frac{1}{2}\right) \ln 2 \\
& -\frac{1}{2 \pi} \kappa a \Theta_{1, \mathrm{r}} \ln \left(2^{\frac{3}{2}-\xi} \sin \pi \xi\right) \\
& =-\frac{1}{2 \pi} \kappa a \Theta_{1, \mathrm{r}} \ln (2 \sin \pi \xi),
\end{aligned}
$$

where we use Euler's reflection formula and Legendre's duplication formula for gamma functions (see Gradshteyn and Ryzhik, 2007):

$$
\begin{aligned}
& \Gamma(\xi) \Gamma\left(\frac{1}{2}-\frac{1}{2} \xi\right) \Gamma\left(1-\frac{1}{2} \xi\right) \\
& =\Gamma(\xi) \Gamma\left(\frac{1}{2}-\frac{1}{2} \xi\right) \Gamma\left(\frac{1}{2}-\frac{1}{2} \xi+\frac{1}{2}\right) \\
& =2^{1-2\left(\frac{1}{2}-\frac{1}{2} \xi\right)} \sqrt{\pi} \Gamma(\xi) \Gamma(1-\xi)=2^{\xi} \pi^{3 / 2} / \sin \pi \xi .
\end{aligned}
$$

Thus, for the symmetric system $(s=0)$, the geometrical jump $\Delta J_{1, \mathrm{r}}^{\text {geom }}$ is equal to half of $J$ in the single potential well. Âs a result, we can renormalize $J$ to cancel $\Delta J_{1, \mathrm{r}}^{\mathrm{geom}}$ : we define $J$ as a half-value of the corresponding variable (i.e. $J \rightarrow J / 2$ ) when particles oscillate in the single well. Section A1 gives $a=1 / g_{c}$, while $\Theta_{1, \mathrm{r}}=4 A_{z_{c}} A_{\Theta}=$ $4\left(A_{z_{c}} z_{c} / s\right) g_{c}=-4 p_{x} g_{c}$. Thus, for $s=0$ we obtain the wellknown expression for the dynamical jump (Timofeev, 1978; Neishtadt, 1986; Cary et al., 1986; Neishtadt, 1987; Büchner and Zelenyi, 1989),

$$
\Delta J^{\mathrm{dyn}}=-\frac{2}{\pi} \kappa p_{x} \ln (2 \sin \pi \xi)
$$
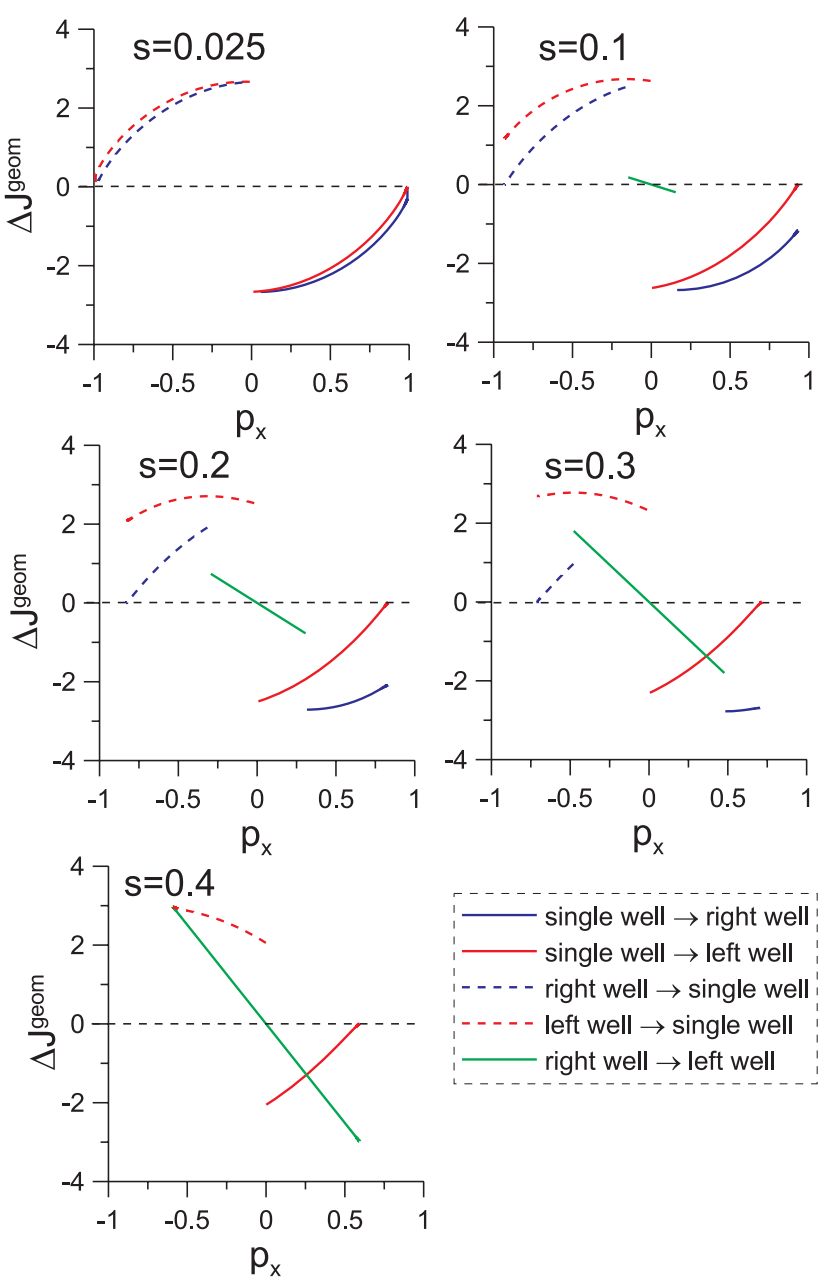

Fig. A1. Geometrical jumps for systems with various $s$.

For asymmetric systems $(s \neq 0)$ we plot $\Delta J^{\text {geom }}$ as functions of the coordinate $p_{x}$ along the uncertainty curve (Fig. A1). One can note that the difference $S_{1}-S_{\mathrm{r}}$ is linearly proportional to $p_{x}$. This dependence can be easily obtained analytically as well.

For the symmetric system $s=0$ the average value of the dynamical jump is equal to zero:

$$
\left\langle\Delta J^{\mathrm{dyn}}\right\rangle_{\xi}=-\frac{2}{\pi} \kappa p_{x} \int_{0}^{1} \ln (2 \sin \pi \xi) d \xi=0 .
$$

For asymmetric systems $(s \neq 0)$ we have the following expressions for the transitions between small wells and the single well (terms $\sim \tilde{u}, \tilde{u}_{\mathrm{r}}$ are equal to zero after averaging over $\xi)$ : 

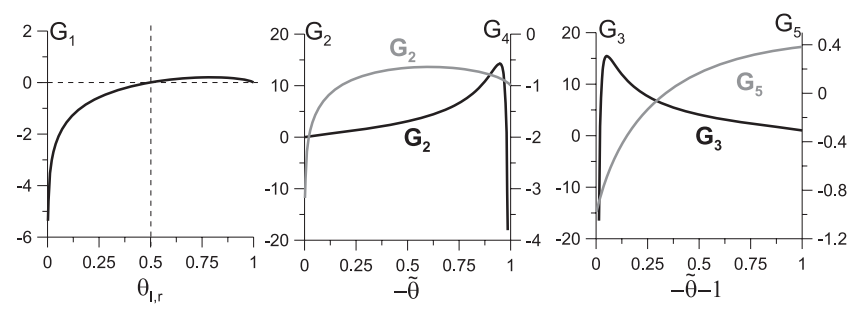

Fig. A2. Functions $G_{i}$ with $i=1 \ldots 5$.

$$
\begin{aligned}
& \left\langle\Delta J^{\mathrm{dyn}}\right\rangle_{\xi}=\kappa a \Theta_{\mathrm{l}, \mathrm{r}} G_{1}\left(\theta_{\mathrm{l}, \mathrm{r}}\right), \quad \Theta_{\mathrm{l}, \mathrm{r}}>0 \\
& \left\langle\Delta J^{\mathrm{dyn}}\right\rangle_{\xi}=\kappa a \Theta_{1} G_{2}(\tilde{\theta})+\frac{1}{2} \kappa\left\{S_{1}, S_{\mathrm{r}}\right\}, \quad \Theta_{1}>0, \Theta_{\mathrm{r}}<0, \Theta>0 \\
& \left\langle\Delta J^{\mathrm{dyn}}\right\rangle_{\xi}=\kappa a \Theta_{1} G_{3}(\tilde{\theta})+\frac{1}{2} \tilde{\theta}^{-2} \kappa\left\{S_{1}, S_{\mathrm{r}}\right\}, \quad \Theta_{1}>0, \Theta_{\mathrm{r}}<0, \Theta<0
\end{aligned}
$$

and for the transition between small wells

$$
\begin{aligned}
& \left\langle\Delta J^{\mathrm{dyn}}\right\rangle_{\xi}=\frac{1}{2} \kappa a\left(\Theta_{\mathrm{r}} \ln \left|\kappa \Theta_{1}\right|-\Theta_{1} \ln \left|\kappa \Theta_{\mathrm{r}}\right|\right) \\
& +\frac{1}{2} \kappa b\left(\Theta_{1}-\Theta_{\mathrm{r}}\right) \\
& -\kappa a \Theta_{1} G_{4}(\tilde{\theta})-\frac{1}{2} \kappa\left\{S_{1}, S_{\mathrm{r}}\right\}, \quad \Theta_{1}>0, \Theta_{\mathrm{r}}<0, \Theta>0 \\
& \left\langle\Delta J^{\mathrm{dyn}}\right\rangle_{\xi}=\frac{1}{2} \kappa a\left(\Theta_{\mathrm{r}} \ln \left|\kappa \Theta_{1}\right|-\Theta_{1} \ln \left|\kappa \Theta_{\mathrm{r}}\right|\right) \\
& +\frac{1}{2} \kappa b\left(\Theta_{1}-\Theta_{\mathrm{r}}\right)-\kappa a \Theta_{1} G_{5}(\tilde{\theta}) \\
& -\frac{1}{2} \tilde{\theta}^{-1} \kappa\left\{S_{1}, S_{\mathrm{r}}\right\}, \quad \Theta_{1}>0, \Theta_{\mathrm{r}}<0, \Theta<0,
\end{aligned}
$$

where

$$
\begin{aligned}
& G_{1}\left(\theta_{\mathrm{l}, \mathrm{r}}\right)=\int_{0}^{1} \ln \frac{(2 \pi)^{3 / 2} d \xi}{\Gamma(\xi) \Gamma\left(\theta_{\mathrm{l}, \mathrm{r}}(1-\xi)\right) \Gamma\left(1-\theta_{\mathrm{l}, \mathrm{r}} \xi\right)} \\
& G_{2}(\tilde{\theta})=\frac{1}{1+\tilde{\theta}} \int_{0}^{1+\tilde{\theta}} \ln \frac{(2 \pi)^{3 / 2} d \xi}{\Gamma(\xi) \Gamma\left(\frac{1}{1+\tilde{\theta}}(1-\xi)\right) \Gamma\left(1-\frac{1}{1+\tilde{\theta}} \xi\right)}, \tilde{\theta}>-1 \\
& G_{3}(\tilde{\theta})=\frac{-1}{1+\tilde{\theta}} \int_{2+\tilde{\theta}}^{1} \ln \frac{(2 \pi)^{3 / 2} d \xi}{\Gamma(\xi) \Gamma\left(\frac{1}{1+\tilde{\theta}}(\xi-1)\right) \Gamma\left(1-\frac{1}{1+\tilde{\theta}} \xi\right)}, \tilde{\theta}<-1 \\
& G_{4}(\tilde{\theta})=\int_{0}^{1} \ln \frac{2 \pi(1-\xi) \sqrt{|\tilde{\theta}|}}{\Gamma(\xi) \Gamma(1+\tilde{\theta}-\tilde{\theta} \xi)} d \xi, \tilde{\theta}>-1 \\
& G_{5}(\tilde{\theta})=\frac{-1}{\tilde{\theta}} \int_{(\tilde{\theta}+1) / \tilde{\theta}}^{1} \ln \frac{2 \pi(1-\xi) \sqrt{|\tilde{\theta}|}}{\Gamma(\xi) \Gamma(1+\tilde{\theta}-\tilde{\theta} \xi)} d \xi, \tilde{\theta}<-1 .
\end{aligned}
$$

Profiles of functions $G_{i}$ are shown in Fig. A2

For several values of $s$ we plot average dynamical jumps $\left\langle\Delta J^{\mathrm{dyn}}\right\rangle_{\xi}$ as functions of the coordinate of uncertainty curve crossing $p_{x}$ in Fig. A3. Here we plot $\left\langle\Delta J^{\text {dyn }}\right\rangle_{\xi}$ without terms $\sim\left\{S_{1}, S_{\mathrm{r}}\right\}$ (analytical expressions for $\left\{S_{1}, S_{\mathrm{r}}\right\}$ can be found in Sect. A3).

\section{A1 Period of fast oscillations}

In this subsection we derive the asymptotic expression for the period of fast oscillations $T_{1, \mathrm{r}}=b_{1, \mathrm{r}}-a \ln |E|$ in the left and
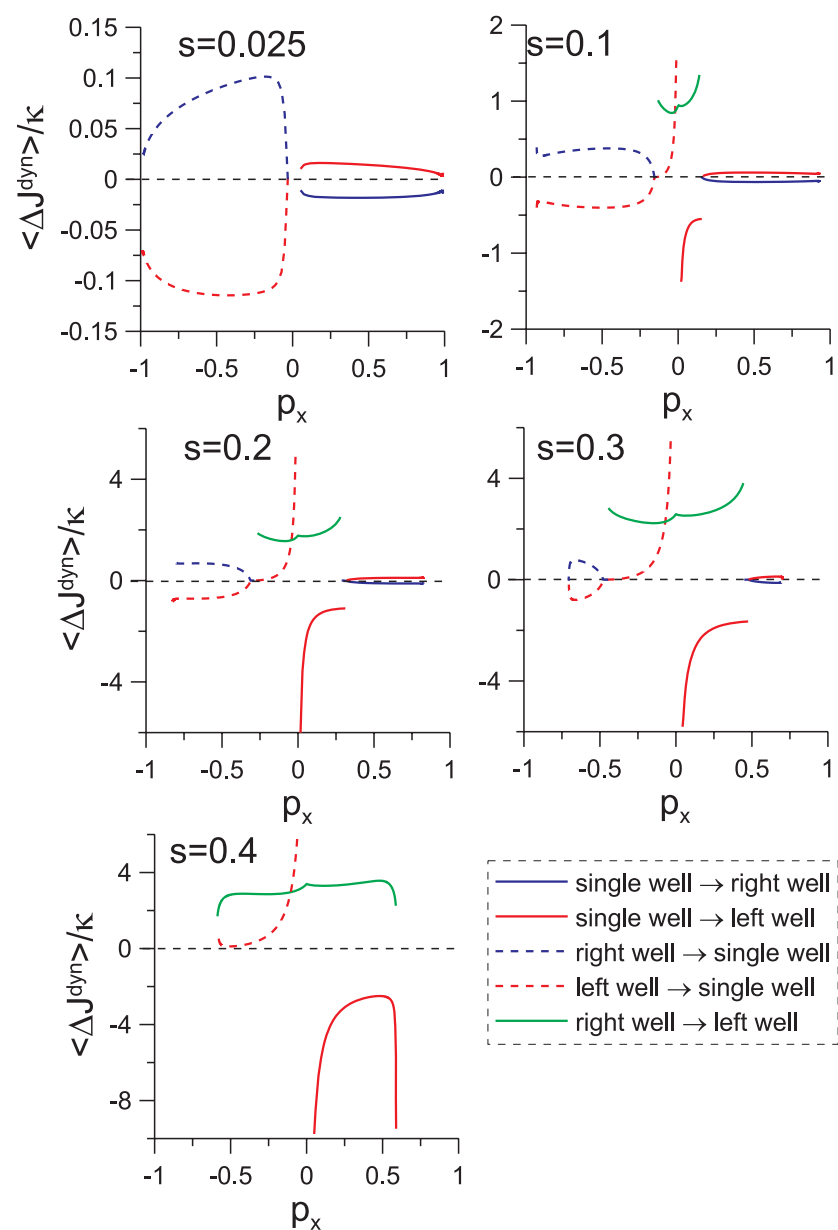

Fig. A3. Dynamical jumps in the system with various $s$ without terms $\sim\left\{S_{1}, S_{\mathrm{r}}\right\}$.

right potential wells. From the general theory it is well known that $T_{1}=T_{\mathrm{r}}$ for Hamiltonian systems like (1) (see Arnold, 1988). Thus, $b_{1}=b_{\mathrm{r}}$, and we can derive the expression only for $T_{\mathrm{r}}$ :

$T_{r}=2 \int_{z^{*}}^{z_{+}} \frac{\mathrm{d} z}{\sqrt{2 H-\left(p_{x}-s z\right)^{2}-\left(\kappa x-\frac{1}{2} z^{2}\right)^{2}}}$,

where $z_{+}$is shown in Fig. A4 and $z^{*}$ corresponds to the leftmost point of the trajectory inside the right separatrix loop. The difference between the $z^{*}$ value and the $z_{c}$ value is determined by the particle energy. We introduce $z_{a}>z^{*}>z_{c}$ and divide the integral $T_{r}$ into two parts $\left(\Delta z=z_{a}-z_{c}>0\right.$ is small enough). The first part of the integral corresponds to integration along the small fragment of the particle trajectory inside the separatrix loop in a close vicinity to $z^{*}$. To perform this integration correctly, $T_{\mathrm{r}}$ should be rewritten as $T_{\mathrm{r}}=\int d p_{z} / \dot{p}_{z}$. However, for Hamiltonian (1) it is well known that integration over $p_{z}$ inside the separatrix loop in the vicinity of $z_{c}$ in the principal approximation gives the 
same result as integration over $z$ outside the separatrix loop. Thus, we can expand the Hamiltonian around $z_{c}$ and write the first part as

$$
\begin{aligned}
& 2 \int_{z_{c}}^{z_{c}+\Delta z} \frac{\mathrm{d} z}{\sqrt{2 H-\left(p_{x}-s z\right)^{2}-\left(\kappa x-\frac{1}{2} z^{2}\right)^{2}}} \\
\approx & 2 \int_{z_{c}}^{z_{c}+\Delta z} \frac{\mathrm{d} z}{\sqrt{2 E+g_{c}^{2}\left(z-z_{c}\right)^{2}}} \\
= & \frac{2}{\sqrt{g}} \ln \left(\frac{g_{c} \Delta z+\sqrt{2 E+g_{c}^{2}(\Delta z)^{2}}}{\sqrt{2 E}}\right) \\
\approx & \frac{\ln \left(4 g_{c}^{2}\right)}{g_{c}}+\frac{2 \ln (\Delta z)}{g_{c}}-\frac{\ln (2 E)}{g_{c}},
\end{aligned}
$$

where we use

$$
\left.\frac{1}{2} \frac{\partial^{2} H}{\partial z^{2}}\right|_{z=z_{c}}=A_{z_{c}}-s^{2}-z_{c}^{2}=g_{c}^{2} .
$$

The second part can be considered as the integral along the separatrix, because we integrate over $\left|z-z_{c}\right|>\Delta z$. In this case we have (see Artemyev et al., 2013)

$$
\begin{aligned}
& 2 H-\left(p_{x}-s z\right)^{2}-\left(\kappa x-\frac{1}{2} z^{2}\right)^{2} \\
& =\left(z-z_{c}\right)\left(A_{z_{c}}-s^{2}-\frac{1}{4}\left(z+z_{c}\right)^{2}\right)
\end{aligned}
$$

and

$$
\begin{aligned}
& \left.2 \int_{z_{c}+\Delta z}^{z_{+}} \frac{\mathrm{d} z}{\left(z-z_{c}\right) \sqrt{A_{z_{c}}-s^{2}-\frac{1}{4}\left(z+z_{c}\right)^{2}}}\right) \\
\approx & \frac{2}{g_{c}} \ln \left(\frac{\left(z_{+}-z_{c}\right)}{\Delta z} \frac{4 g_{c}^{2}}{2\left(g_{c}^{2}+z_{c}^{2}\right)-z_{c}\left(z_{+}+z_{c}\right)}\right) \\
= & -\frac{2 \ln (\Delta z)}{g_{c}}+\frac{2 \ln \left(4 g_{c}^{2}\right)}{g_{c}}-\frac{\ln \left(g_{c}^{2}+z_{c}^{2}\right)}{g_{c}},
\end{aligned}
$$

where we take into account that $z_{ \pm}= \pm 2 \sqrt{g_{c}^{2}+z_{c}^{2}}-z_{c}$. The final expression for the period is

$T_{1, \mathrm{r}}=-\frac{\ln (2 E)}{g_{c}}+\frac{3 \ln \left(4 g_{c}^{2}\right)}{g_{c}}-\frac{\ln \left(g_{c}^{2}+z_{c}^{2}\right)}{g_{c}}$.

Therefore, we have

$$
a=1 / g_{c}
$$

$b_{1}=b_{\mathrm{r}}=\frac{6 \ln \left(2 g_{c}\right)}{g_{c}}-\frac{\ln \left(g_{c}^{2}+z_{c}^{2}\right)}{g_{c}}-\frac{\ln (2)}{g_{c}}$.

\section{A2 The improved quasi-adiabatic invariant}

In this section we derive the asymptotic expression for the improved quasi-adiabatic invariant $J=I_{z}+\kappa u$ for $p_{z}=0$, where $u$ is defined as (see Neishtadt, 1987)

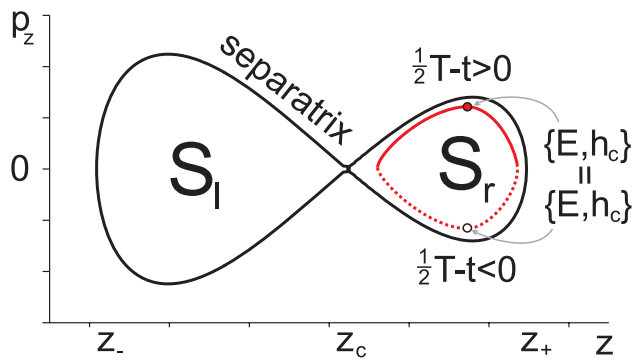

Fig. A4. Schematic presentation of calculation of the integral $\int(T / 2-t)\left\{E, h_{c}\right\} \mathrm{d} t$.

$$
\begin{aligned}
u= & \frac{1}{4 \pi}\left[\int_{0}^{T}\left(\frac{\partial E}{\partial p_{x}} \int_{0}^{t} \frac{\partial E}{\partial \kappa x} \mathrm{~d} t^{\prime}\right) \mathrm{d} t-\int_{0}^{T}\left(\frac{\partial E}{\partial \kappa x} \int_{0}^{t} \frac{\partial E}{\partial p_{x}} \mathrm{~d} t^{\prime}\right) \mathrm{d} t\right] \\
& +\frac{1}{2 \pi} \int_{0}^{T}\left(\frac{T}{2}-t\right)\left(\frac{\partial h_{c}}{\partial p_{x}} \frac{\partial E}{\partial \kappa x}-\frac{\partial h_{c}}{\partial \kappa x} \frac{\partial E}{\partial p_{x}}\right) \mathrm{d} t .
\end{aligned}
$$

Here $T\left(\kappa x, p_{x}\right)$ is the period of fast motion. Integration is performed along a trajectory with a certain initial point in the plane $\left(z, p_{z}\right)$. We choose this point as $p_{z}=0$, and as a result, the lower limit of integration $t=0$ corresponds to the starting point where the trajectory crosses the $p_{z}$ axis (the leftmost point of the trajectory). We consider one of the two potential wells (the right one). The last term of $u$ can be written as

$$
\frac{1}{2 \pi} \int_{0}^{T}\left(\frac{1}{2} T-t\right)\left\{E, h_{c}\right\} \mathrm{d} t,
$$

where $\{\ldots\}$ is the Poisson bracket. We divide this integral into two parts: $t \in[0, T / 2]$ and $t \in[T / 2, T]$. The expression $\left\{E, h_{c}\right\}$ does not depend on $p_{z}$, and thus it has the same values for $t$ and $t+T / 2$. Expression $T / 2-t=-(t-T / 2)$ is positive for $t \in[0, T / 2]$ and negative for $t \in[T / 2, T]$. As a result, the total integral is equal to zero (see the scheme in Fig. A4).

Now we consider the first two integrals in the expression for $u$ for the right well. The inner integrals are

$$
\begin{aligned}
& \int_{0}^{t} \frac{\partial E}{\partial \kappa x} \mathrm{~d} t^{\prime}=-\frac{1}{2}\left\{\begin{array}{l}
\Im_{\Sigma}^{\left\{z_{c}, z\right\}}, t<\frac{1}{2} T \\
\left.\Im_{\Sigma}^{\left.\mid z_{c}, z_{+}\right\}}+\Im_{\Sigma} z_{\Sigma}, z_{+}\right\}
\end{array}=-\frac{1}{2} \Im_{\Sigma}^{\left\{z_{c}, z_{+}\right\}} \pm \frac{1}{2} \Im_{\Sigma}^{\left\{z, z_{+}\right\}}\right. \\
& \int_{0}^{t} \frac{\partial E}{\partial p_{x}} \mathrm{~d} t^{\prime}=-s\left\{\begin{array}{l}
\Im_{0}^{\left\{z_{c}, z\right\}}, t<\frac{1}{2} T \\
\Im_{0}^{\left\{z_{c}, z_{+}\right\}}+\Im_{0}^{\left\{z, z_{+}\right\}}=-s \Im_{0}^{\left\{z_{c}, z_{+}\right\}} \pm s \Im_{0}^{\left\{z, z_{+}\right\}}
\end{array}\right.
\end{aligned}
$$

where \pm corresponds to $t<T / 2$ and $t>T / 2$, and we use $p_{z}^{2}=2\left(H-h_{c}\right)=\left(z-z_{c}\right)^{2} g^{2}(z)$ (see Artemyev et al., 2013). Integrals $\Im$ are given in Table A1. 
Table A1. Integrals $\mathfrak{\Im}_{n}^{\left\{z_{1}, z_{2}\right\}}=\int_{z_{1}}^{z_{2}}\left(z^{n} / g(z)\right) \mathrm{d} z$ and $\mathfrak{\Im}_{\Sigma}^{\left\{z_{1}, z_{2}\right\}}=$ $\Im_{1}^{\left\{z_{1}, z_{2}\right\}}+z_{c} \Im_{0}^{\left\{z_{1}, z_{2}\right\}}$.

\begin{tabular}{lc}
\hline integral & value \\
\hline $\mathfrak{J}_{\sum}^{\left\{z_{c}, z_{+}\right\}}$ & $4 g_{c}$ \\
$\mathfrak{J}_{\sum}^{\left\{z, z_{+}\right\}}$ & $4 g(z)$ \\
$\mathfrak{J}_{0}^{\left\{z_{c}, z_{+}\right\}}$ & $\pi-2 \arctan \left(\frac{z_{c}}{g_{c}}\right)$ \\
$\mathfrak{\Im}_{0}^{\left\{z, z_{+}\right\}}$ & $\pi-2 \arctan \left(\frac{z_{c}+z}{2 g_{c}}\right)$ \\
\hline
\end{tabular}

The first integral in expression for $u$ is

$\int_{0}^{T}\left(\frac{\partial E}{\partial p_{x}} \int_{0}^{t} \frac{\partial E}{\partial \kappa x} \mathrm{~d} t^{\prime}\right) \mathrm{d} t$
$=4 s g_{c} \Im_{0}^{\left\{z_{c}, z_{+}\right\}}=8 s g_{c}\left(\frac{\pi}{2}-\arctan \left(\frac{z_{c}}{g_{c}}\right)\right)$,

and the second integral is

$\int_{0}^{T}\left(\frac{\partial E}{\partial \kappa x} \int_{0}^{t} \frac{\partial E}{\partial p_{x}} \mathrm{~d} t^{\prime}\right) \mathrm{d} t=s \Im_{\Sigma}^{\left\{z_{c}, z_{+}\right\}}\left(\pi-2 \arctan \left(\frac{z_{c}}{g_{c}}\right)\right)=$ $=4 s \pi g_{c}-8 s g_{c} \arctan \left(\frac{z_{c}}{g_{c}}\right)$.

Then, the difference of the first and the second terms is equal to zero, and $u=0$ for the right potential well (at $\left.p_{z}=0\right)$. The same conclusion is valid for the left well and for the single well. Moreover, one can show that $\langle u\rangle_{\xi}=0$ (see details in Neishtadt, 1987).

\section{A3 Calculation of $\left\{S_{\mathrm{I}, \mathrm{r}}, S\right\}$}

Here we derive the expression for $\left\{S_{1, \mathrm{r}}, S\right\}$. Due to $S=S_{1}+S_{\mathrm{r}}$ we need to obtain the expression $\left\{S_{1}, S_{\mathrm{r}}\right\}$, where

$S_{1, \mathrm{r}}=2 \int_{z_{\mathrm{l}, \mathrm{r}}}^{z_{1, \mathrm{r}}^{\max }} \sqrt{2 U\left(\kappa x, p_{x}, z_{c}\right)-2 U\left(\kappa x, p_{x}, z\right)} \mathrm{d} z$

and $z_{1, \mathrm{r}}^{\min }=z_{-}, z_{c}, z_{1, \mathrm{r}}^{\max }=z_{c}, z_{+}, 2 U=2 H-p_{z}^{2}$ (see Artemyev et al., 2013). We can write

$$
\begin{aligned}
& 2 U\left(\kappa x, p_{x}, z_{c}\right)-2 U\left(\kappa x, p_{x}, z\right)= \\
& \left(p_{x}-s z_{c}\right)^{2}+\left(\kappa x-\frac{1}{2} z_{c}^{2}\right)^{2}-\left(p_{x}-s z\right)^{2}-\left(\kappa x-\frac{1}{2} z^{2}\right)^{2} \\
& =-2 s p_{x}\left(z_{c}-z\right)-\kappa x\left(z_{c}^{2}-z^{2}\right)+s^{2}\left(z_{c}^{2}-z^{2}\right)+\frac{1}{4}\left(z_{c}^{4}-z^{4}\right) .
\end{aligned}
$$

Then we have

$$
\begin{aligned}
& \frac{\partial S_{1, \mathrm{r}}}{\partial \kappa x}=-\int_{z_{1, \mathrm{r}}}^{z_{1, \mathrm{r}}^{\max }} \frac{z_{c}^{2}-z^{2}}{\sqrt{2 U\left(z_{c}\right)-2 U(z)}} \mathrm{d} z+\frac{\partial S_{1, \mathrm{r}}}{\partial z_{c}} \frac{\partial z_{c}}{\partial \kappa x} \\
& \frac{\partial S_{1, \mathrm{r}}}{\partial p_{x}}=-2 s \int_{z_{\mathrm{min}}^{\min }}^{z_{1, \mathrm{r}}^{\max }} \frac{z_{c}-z}{\sqrt{2 U\left(z_{c}\right)-2 U(z)}} \mathrm{d} z+\frac{\partial S_{1, \mathrm{r}}}{\partial z_{c}} \frac{\partial z_{c}}{\partial p_{x}},
\end{aligned}
$$

where we can use expressions for $\kappa x=(1 / 2) z_{c}^{2}+A_{z_{c}}$ and $p_{x}=\left(z_{c} / s\right)\left(s^{2}-A_{z_{c}}\right)$ with $A_{z_{c}}=\left(1+z_{c}^{2} / s^{2}\right)^{-1 / 2}$ (see Artemyev et al., 2013). The corresponding integrals are

$$
\begin{aligned}
& \int_{z_{1, \mathrm{r}}}^{z_{1, \mathrm{r}}^{\max }} \frac{z_{c}^{2}-z^{2}}{\sqrt{2 U\left(z_{c}\right)-2 U(z)}} \mathrm{d} z=4 g_{c} \\
& \int_{z_{\mathrm{min}}}^{\max } \frac{z_{c}-z}{\sqrt{2 U\left(z_{c}\right)-2 U(z)}} \mathrm{d} z=\pi \pm 2 \arctan \left(\frac{z_{c}}{g_{c}}\right) .
\end{aligned}
$$

Derivatives $\partial S_{1, \mathrm{r}} / \partial z_{c} \sim \partial U\left(z_{c}\right) / \partial z_{c}$ are equal to zero due to the definition of $z_{c}$. Thus, finally we have

$$
\frac{\partial S_{1}}{\partial \kappa x} \frac{\partial S_{\mathrm{r}}}{\partial p_{x}}-\frac{\partial S_{1}}{\partial p_{x}} \frac{\partial S_{\mathrm{r}}}{\partial \kappa x}=32 s g_{c} \arctan \left(\frac{z_{c}}{g_{c}}\right) .
$$

\section{Appendix B}

\section{Resonances}

In this appendix we describe the effect of resonant interaction of a particle with the CS. In the course of interaction with the CS, particles cross the uncertainty curve (and the separatrix) twice: when particles approach the neutral plane moving along the field lines, and when they leave the neutral plane after a half-turn around $B_{z}$. Therefore, there are two dynamical jumps of the quasi-adiabatic invariant.

For the symmetric system $(s=0)$ we have the expression for the dynamical jump $\Delta J^{\mathrm{dyn}}=-(2 / \pi) \kappa p_{x} \ln (2 \sin \xi \pi)$, where $\xi \in(0,1)$ is a random value (see Appendix A). Thus, the sum of two successive jumps is

$$
\sum \Delta J^{\mathrm{dyn}}=-\frac{2}{\pi} \kappa p_{x} \ln \left(\frac{2 \sin \pi \xi}{2 \sin \pi(\xi+\Delta \xi)}\right),
$$

where $\Delta \xi$ is a difference of phases between two separatrix crossings. The full expression for $\Delta \xi$ can be found in Neishtadt and Vasiliev (2005): $\Delta \xi+\xi=\operatorname{Frac}(W-\xi)$, where

$$
W=\frac{1}{\kappa \pi} \int_{\kappa \tilde{x}}^{\kappa x^{*}} \frac{\Omega_{z}}{p_{x}} d \kappa x
$$

and $\kappa \tilde{x}$ is determined as the $\kappa x$ value at the point where a trajectory $I_{z}\left(\kappa x, p_{x}\right)=$ const crosses the $p_{x}=0$ axis. Here 

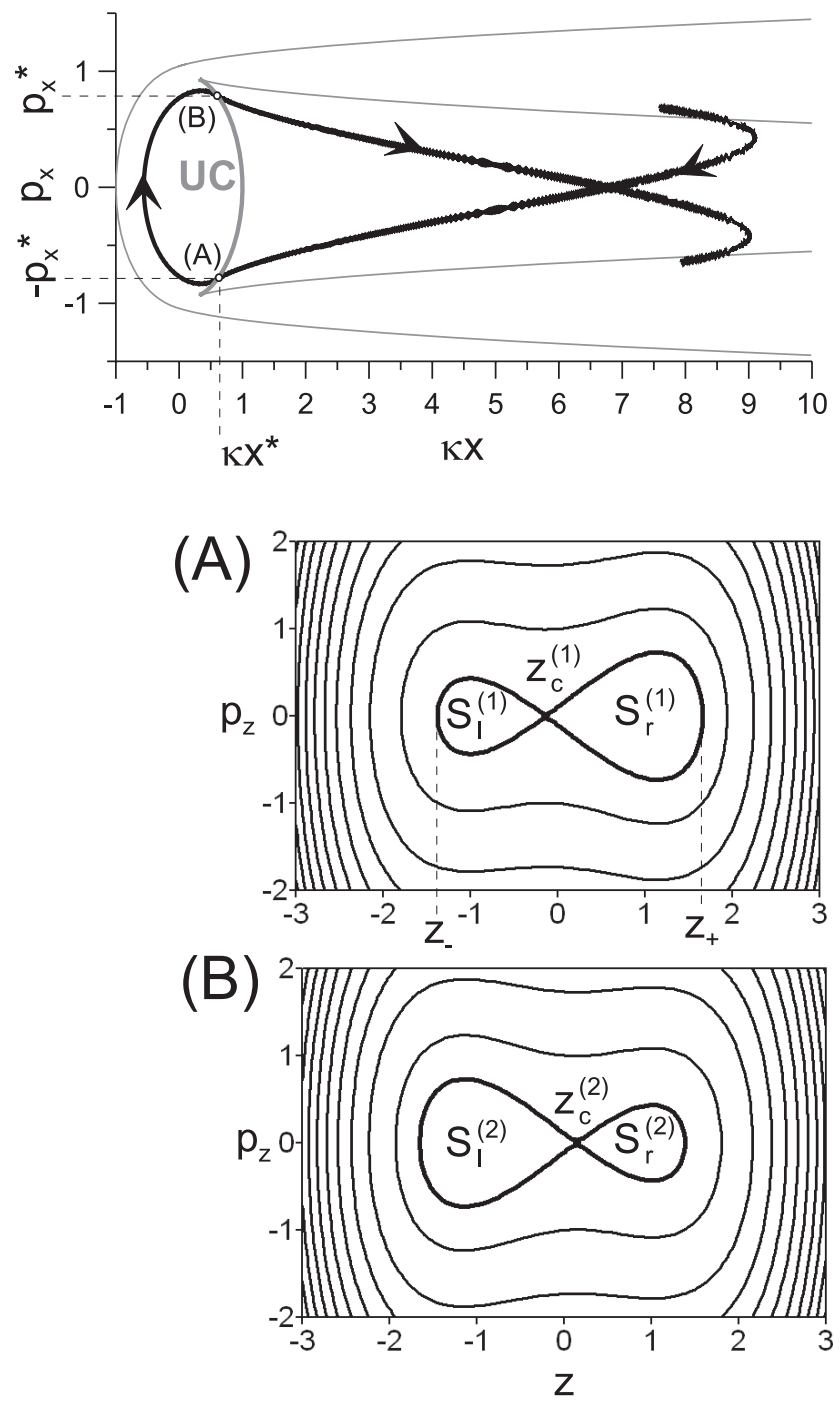

Fig. B1. Schematical view of a particle trajectory with two crossings of the uncertainty curve.

$\operatorname{Frac}(\cdot)$ denotes the fractional part of a number in brackets $(\cdot), \kappa x^{*}$ is the coordinate of uncertainty curve crossings and $\Omega_{z}(\kappa x)$ is the frequency of fast oscillations:

$\Omega_{z}=2 \pi\left(\oint \mathrm{d} z / p_{z}\right)^{-1}$.

If $\Delta \xi$ is equal to $\pi-2 \xi$ (i.e. $W=\pi$ ), we have $\sum \Delta J^{\text {dyn }}=$ 0 . This condition corresponds to the equation $W=\pi$, which is independent of $\xi$. Thus, the condition $\sum \Delta J^{\text {dyn }}=0$ can be simultaneously satisfied for a large particle population. The equation $W=\pi$ can be solved with regard to $\kappa$, and corresponding solutions are called resonant $\kappa$ values (Büchner and Zelenyi, 1989; Zelenyi et al., 2013).

To investigate the same effect of the resonance for systems with $s \neq 0$, we write equations of the uncertainty curve in the $\left(\kappa x, p_{x}\right)$ plane (see Artemyev et al., 2013)
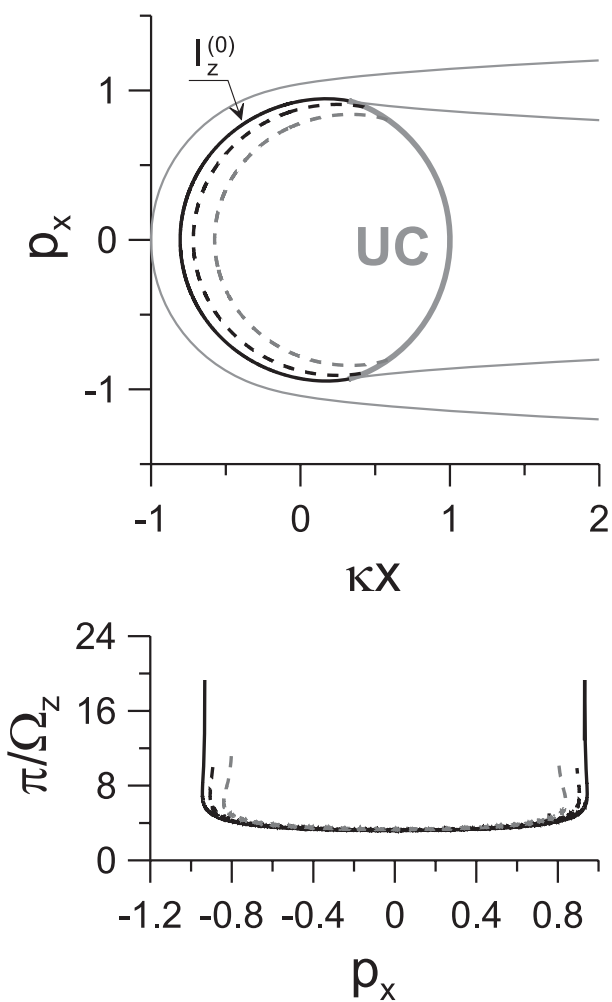

Fig. B2. Fragments of three trajectories and corresponding dependence of $\pi / \Omega_{z}$ on $p_{x}$.

$p_{x}=\left(z_{c} / s\right)\left(s^{2}-A_{z_{c}}\right)$
$\kappa x=\frac{1}{2} z_{c}^{2}+A_{z_{c}}$.

One can see that the sign of $p_{x}$ is defined by the sign of $z_{c}$ (because $A_{z_{c}}-s^{2}>0$ for entire range of $z_{c}$ ). Due to the symmetry of the phase portrait of the system in the plane $\left(\kappa x, p_{x}\right)$ relative to $p_{x}=0$, particles that crossed the uncertainty curve at $p_{x}=-p_{x}^{*}<0$ should cross it again at $p_{x}=p_{x}^{*}>0$. A schematic view of such a trajectory is presented in Fig. B1. We consider the particles that come to the uncertainty curve inside the right well and after the second crossing are captured in the left well. These particles can return to the initial coordinate $\kappa x$ in the opposite side relative to the neutral plane $z=0$. For these particles the coordinate of the saddle point in the second crossing $z_{c}^{(2)}$ is equal to $-z_{c}^{(1)}$, where $z_{c}^{(1)}$ is the coordinate of the saddle point in the first crossing. Because $S_{r}\left(z_{c}\right)=S_{1}\left(-z_{c}\right)$ (see expression for $S_{\mathrm{l}, \mathrm{r}}$ in Appendix A), we have $S_{\mathrm{r}}^{(1)}=S_{1}^{(2)}$. Thus, two successive geometrical jumps compensate each other for such trajectories.

The rates $\kappa \Theta_{1, \mathrm{r}}$ of evolution of areas can be presented as a sum $\Theta_{1, \mathrm{r}}=4 A_{z} A_{\Theta} \pm 2 \pi s A_{z_{c}}$. Thus, we have $\theta=\theta_{\mathrm{r}}^{(1)}=\theta_{1}^{(2)}$. Dynamical jumps for two crossings are 

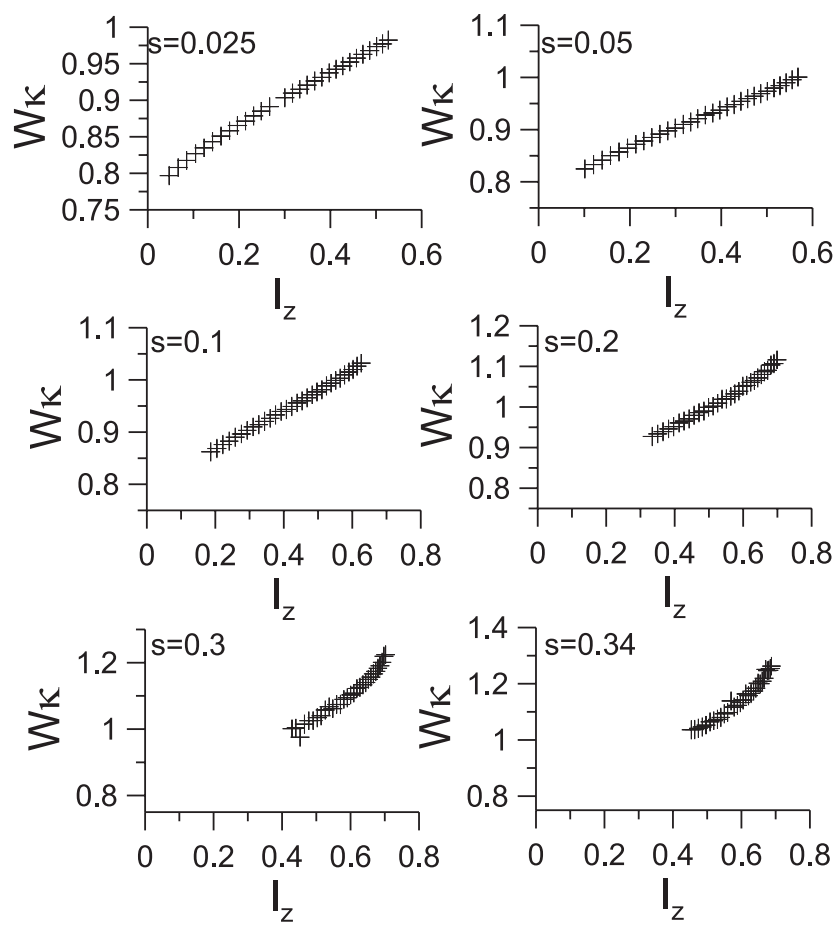

Fig. B3. $W$ as a function of $I_{z}$ for various $s$ (to substitute values of $W$ in the equation for dynamical jumps, one should take $\operatorname{Frac}(W / \kappa))$.

$$
\begin{aligned}
\Delta J_{(1,2)}^{\mathrm{dyn}} & = \pm \frac{\kappa \Theta_{\mathrm{r}}^{(1)}}{2 \pi}\left(\xi^{(1,2)}-\frac{1}{2}\right)\left(a \ln \frac{\kappa \Theta_{\mathrm{r}}^{(1)}}{(\kappa \Theta)^{2 \theta}}-b(1-2 \theta)\right) \\
& \mp \frac{\kappa a \Theta_{\mathrm{r}}^{(1)}}{2 \pi} \ln \frac{(2 \pi)^{3 / 2}}{\Gamma\left(\xi^{(1,2)}\right) \Gamma\left(\theta\left(1-\xi^{(1,2)}\right) \Gamma\left(1-\theta \xi^{(1,2)}\right)\right.},
\end{aligned}
$$

where $a=a\left(z_{c}\right)=a\left(-z_{c}\right)$ and $b=b\left(z_{c}\right)=b\left(-z_{c}\right)$ (see Sect. A1). The sum of these jumps gives

$$
\begin{gathered}
\sum \Delta J^{\text {dyn }}=\frac{\kappa \Theta_{\mathrm{r}}^{(1)}}{2 \pi} \Delta \xi\left(a \ln \frac{\kappa \Theta_{\mathrm{r}}^{(1)}}{(\kappa \Theta)^{2 \theta}}-b(1-2 \theta)\right) \\
-\frac{\kappa a \Theta_{\mathrm{r}}^{(1)}}{2 \pi} \ln \frac{\Gamma\left(\xi^{(2)}\right) \Gamma\left(\theta\left(1-\xi^{(2)}\right)\right) \Gamma\left(1-\theta \xi^{(2)}\right)}{\Gamma\left(\xi^{(1)}\right) \Gamma\left(\theta\left(1-\xi^{(1)}\right)\right) \Gamma\left(1-\theta \xi^{(1)}\right)},
\end{gathered}
$$

where $\Delta \xi=\xi^{(2)}-\xi^{(1)}$. One can see that the condition $\Delta \xi=$ const $-2 \xi^{(1)}$ does not obviously result in $\sum \Delta J^{\text {dyn }}=$ 0 . Thus, the resonant condition $\sum \Delta J^{\text {dyn }}=0$ corresponds to a certain equation, which depends on $\xi^{(1)}$ and on coordinates of the uncertainty curve crossing. Such a condition cannot be satisfied simultaneously for a large particle population. This is the first effect, which results in the destruction of resonances.

Let us consider the second effect, which is responsible for destruction of resonances. This effect corresponds to dependence of the frequency $\Omega_{z}$ on the quasi-adiabatic invariant $I_{z}$ :

$$
\begin{aligned}
& \oint \mathrm{d} z / p_{z}=2 \int_{z-}^{z_{+}} \frac{\mathrm{d} z}{\sqrt{2 H-\left(p_{x}-s z\right)^{2}-\left(\kappa x-\frac{1}{2} z^{2}\right)^{2}}} \\
& I_{z}=2 \int_{z_{-}}^{z_{+}} \sqrt{2 H-\left(p_{x}-s z\right)^{2}-\left(\kappa x-\frac{1}{2} z^{2}\right)^{2}} \mathrm{~d} z,
\end{aligned}
$$

where $z_{ \pm}$are shown in Fig. B1. Therefore, for each $I_{z}$ we have a certain $\Omega_{z}(\kappa x)$ and can calculate the corresponding integral $W$. Examples of three trajectories and corresponding distributions of $\Omega_{z}$ along the trajectories are shown in Fig. B2. At the vicinity of the uncertainty curve the frequency $\Omega_{z}$ tends to zero. This is an effect of the logarithmic singularity of the frequency of particle oscillations near the separatrix.

We calculate $W$ for $I_{z} \geq I_{z}^{(0)}$, where $I_{z}^{(0)}$ corresponds to the trajectory crossing the uncertainty curve at the endpoints (see Fig. B2). Corresponding dependencies of $W$ on $I_{z}$ are shown in Fig. B3. One can see that for small $s$ the function $W\left(I_{z}\right)$ tends to $0.76 / \kappa$ as $I_{z}^{(0)} \rightarrow 0$ (this value can be calculated analytically; see Büchner and Zelenyi, 1989).

For small $s$ the derivative $\partial W / \partial I_{z}$ is small enough. Thus, if a value of $\kappa$ in the system is suitable to obtain the resonant value of $\Delta \xi$ for $I_{z}=I_{z}^{(0)}$, then for particles with other $I_{z}$, the corresponding $\Delta \xi$ should have similar values. As a result, the resonant condition (condition for $\kappa$ ) is satisfied for particles with various $I_{z}$ (see discussion in Büchner and Zelenyi, 1989).

Increasing $s$ results in the increase of the derivative $\partial W / \partial I_{z}$. Thus, $\Delta \xi$ changes more substantially with $I_{z}$ for $s>0$. It means that even if $\kappa$ has a suitable (resonant) value to obtain the resonant value of $\Delta \xi$ for $I_{z}=I_{z}^{(0)}$, for particles with other values of $I_{z}$, the corresponding $\Delta \xi \sim$ $\left(\partial W / \partial I_{z}\right)\left(I_{z}-I_{z}^{(0)}\right)$ should be far from the resonant value. This results in a decrease in the number of particles for which the resonant condition is satisfied for the same $\kappa$.

Acknowledgements. We are very grateful to A. A. Vasiliev for useful discussion. This work was supported in part by the RF Presidential Program for the State Support of Leading Scientific Schools (project NSh-2519.2012.1) and the Russian Foundation for Basic Research (projects 11-02-01166, 13-01-00251).

Edited by: J. Büchner

Reviewed by: H. Karimabadi and A. Anastasiadis

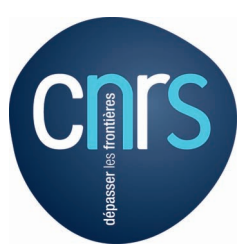

The publication of this article is financed by CNRS-INSU. 


\section{References}

Anastasiadis, A., Gontikakis, C., and Efthymiopoulos, C.: Particle Interactions with single or multiple 3D solar reconnecting current sheets, Solar Phys., 253, 199-214, doi:10.1007/s11207-0089234-4, 2008.

Angelopoulos, V., Kennel, C. F., Coroniti, F. V., Pellat, R., Spence, H. E., Kivelson, M. G., Walker, R. J., Baumjohann, W., Feldman, W. C., and Gosling, J. T.: Characteristics of ion flow in the quiet state of the inner plasma sheet, Geophys. Res. Lett., 20, 17111714, doi:10.1029/93GL00847, 1993.

Arnold, V. I.: Geometrical methods in the theory of ordinary differential equations, Springer-Verlag, New York, 1988.

Arnold, V. I., Kozlov, V. V., and Neishtadt, A. I.: Mathematical aspects of classical and celestial mechanics, dynamical systems III. encyclopedia of mathematical sciences, Springer-Verlag, New York, 3rd Edn., 2006.

Artemyev, A. V.: A model of one-dimensional current sheet with parallel currents and normal component of magnetic field, Phys. Plasmas, 18, 022104, doi:10.1063/1.3552141, 2011.

Artemyev, A. V. and Zelenyi, L. M.: Kinetic structure of current sheets in the Earth magnetotail, Space Sci. Rev., doi:10.1007/s11214-012-9954-5, in press, 2013.

Artemyev, A. V. and Zimovets, I.: Stability of current sheets in the solar corona, Solar Phys., 277, 283-298, doi:10.1007/s11207011-9908-1, 2012.

Artemyev, A. V., Neishtadt, A. I., and Zelenyi, L. M.: Ion motion in the current sheet with sheared magnetic field - Part 1: Quasi-adiabatic theory, Nonlin. Processes Geophys., 20, 163 178, doi:10.5194/npg-20-163-2013, 2013.

Ashour-Abdalla, M., Zelenyi, L. M., Bosqued, J. M., and Kovrazhkin, R. A.: Precipitation of fast ion beams from the plasma sheet boundary layer, Geophys. Res. Lett., 19, 617-620, doi:10.1029/92GL00048, 1992.

Ashour-Abdalla, M., Berchem, J. P., Büchner, J., and Zelenyi, L. M.: Shaping of the magnetotail from the mantle Global and local structuring, J. Geophys. Res., 98, 5651-5676, doi:10.1029/92JA01662, 1993.

Ashour-Abdalla, M., Zelenyi, L. M., Peroomian, V., and Richard, R. L.: Consequences of magnetotail ion dynamics, J. Geophys. Res., 99, 14891-14916, doi:10.1029/94JA00141, 1994.

Baek, S.-C., Choi, D.-I., and Horton, W.: Dawn-dusk magnetic field effects on ions accelerated in the current sheet, J. Geophys. Res., 100, 14935-14942, doi:10.1029/95JA01610, 1995.

Baumjohann, W., Roux, A., Le Contel, O., Nakamura, R., Birn, J., Hoshino, M., Lui, A. T. Y., Owen, C. J., Sauvaud, J.-A., Vaivads, A., Fontaine, D., and Runov, A.: Dynamics of thin current sheets: Cluster observations, Ann. Geophys., 25, 13651389, doi:10.5194/angeo-25-1365-2007, 2007.

Birmingham, T. J.: Pitch angle diffusion in the Jovian magnetodisc, J. Geophys. Res., 89, 2699-2707, doi:10.1029/JA089iA05p02699, 1984.

Büchner, J.: Correlation-modulated chaotic scattering in the earth's magnetosphere, Geophys. Res. Lett., 18, 1595-1598, doi:10.1029/91GL01905, 1991.

Büchner, J. and Zelenyi, L. M.: Deterministic chaos in the dynamics of charged particles near a magnetic field reversal, Phys. Lett. A, 118, 395-399, doi:10.1016/0375-9601(86)90268-9, 1986.

Büchner, J. and Zelenyi, L. M.: Regular and chaotic charged particle motion in magnetotaillike field reversals. I - Basic the- ory of trapped motion, J. Geophys. Res., 94, 11821-11842, doi:10.1029/JA094iA09p11821, 1989.

Büchner, J. and Zelenyi, L. M.: The separatrix tentacle effect of ion acceleration to the plasma sheet boundary, Geophys. Res. Lett., 17, 127-130, doi:10.1029/GL017i002p00127, 1990.

Büchner, J. and Zelenyi, L. M.: Regular and chaotic particle motion in sheared magnetic field reversals, Adv. Space Res., 11, 177182, doi:10.1016/0273-1177(91)90030-N, 1991.

Burkhart, G. R. and Chen, J.: Differential memory in the earth's magnetotail, J. Geophys. Res., 96, 14033, doi:10.1029/91JA01137, 1991.

Burkhart, G. R., Drake, J. F., Dusenbery, P. B., and Speiser, T. W.: A particle model for magnetotail neutral sheet equilibria, J. Geophys. Res., 97, 13799-13815, doi:10.1029/92JA00495, 1992.

Burkhart, G. R., Dusenbery, P. B., and Speiser, T. W.: Particle chaos and pitch angle scattering, J. Geophys. Res., 100, 107-118, doi:10.1029/94JA02221, 1995.

Carati, A., Zuin, M., Maiocchi, A., Marino, M., Martines, E., and Galgani, L.: Transition from order to chaos, and density limit, in magnetized plasmas, Chaos, 22, 033124, doi:10.1063/1.4745851, 2012.

Cary, J. R., Escande, D. F., and Tennyson, J. L.: Adiabatic-invariant change due to separatrix crossing, Phys. Rev. A, 34, 4256-4275, 1986.

Chapman, S. C. and Rowlands, G.: Are particles detrapped by constant $B_{y}$ in static magnetic reversals?, J. Geophys. Res., 103, 4597-4604, doi:10.1029/97JA01737, 1998.

Chen, J.: Nonlinear dynamics of charged particles in the magnetotail, J. Geophys. Res., 97, 15011, doi:10.1029/92JA00955, 1992.

Chen, J. and Palmadesso, P. J.: Chaos and nonlinear dynamics of single-particle orbits in a magnetotaillike magnetic field, J. Geophys. Res., 91, 1499-1508, doi:10.1029/JA091iA02p01499, 1986.

Cheng, A. F. and Decker, R. B.: Nonadiabatic particle motion and corotation lag in the Jovian magnetodisk, J. Geophys. Res., 97, 1397-1402, doi:10.1029/91JA02407, 1992.

Chirikov, B. V.: A universal instability of many-dimensional oscillator systems, Physics Reports, 52, 263-379, doi:10.1016/03701573(79)90023-1, 1979.

Chirikov, B. V.: Particle dynamics in magnetic traps, vol. 13, Consultants Bureau, New York, 1st Edn., 1987.

Cowley, S. W. H.: Magnetospheric asymmetries associated with the y-component of the IMF, Plan. Sp. Sci., 29, 79-96, doi:10.1016/0032-0633(81)90141-0, 1981.

Delcourt, D. C., Martin, Jr., R. F., and Alem, F.: A simple model of magnetic moment scattering in a field reversal, Geophys. Res. Lett., 21, 1543-1546, doi:10.1029/94GL01291, 1994.

Delcourt, D. C., Zelenyi, L. M., and Sauvaud, J.-A.: Magnetic moment scattering in a field reversal with nonzero $B_{y}$ component, J. Geophys. Res., 105, 349-360, doi:10.1029/1999JA900451, 2000.

Delcourt, D. C., Grimald, S., Leblanc, F., Berthelier, J.-J., Millilo, A., Mura, A., Orsini, S., and Moore, T. E.: A quantitative model of the planetary Na+ contribution to Mercury's magnetosphere, Ann. Geophys., 21, 1723-1736, doi:10.5194/angeo-211723-2003, 2003.

Dolgonosov, M. S., Zimbardo, G., and Greco, A.: Influence of the electric field perpendicular to the current sheet on ion 
beamlets in the magnetotail, J. Geophys. Res., 115, A02209, doi:10.1029/2009JA014398, 2010.

Eastwood, J. W.: Consistency of fields and particle motion in the 'Speiser' model of the current sheet, Planet. Space Sci., 20, 1555-1568, doi:10.1016/0032-0633(72)90182-1, 1972.

Galeev, A. A., Kuznetsova, M. M., and Zelenyi, L. M.: Magnetopause stability threshold for patchy reconnection, Space Sci. Rev., 44, 1-41, doi:10.1007/BF00227227, 1986.

Gradshteyn, I. S. and Ryzhik, I. M.: Table of Integrals, Series, and Products, Academic Press, 2007.

Greco, A., Veltri, P., Zimbardo, G., Taktakishvilli, A. L., and Zelenyi, L. M.: Numerical simulation of ion dynamics in the magnetotail magnetic turbulence: On collisionless conductivity, Nonlin. Processes Geophys., 7, 159-166, doi:10.5194/npg-7-1592000, 2000.

Grigorenko, E. E., Fedorov, A. O., Budnik, E. Y., Sauvaud, J.-A., Zelenyi, L. M., Reme, H., and Dunlop, M. W.: Spatial structure of beamlets according to Cluster observations, Planet. Space Sci., 53, 245-254, doi:10.1016/j.pss.2004.09.050, 2005.

Grigorenko, E. E., Zelenyi, L. M., Dolgonosov, M. S., Artemiev, A. V., Owen, C. J., Sauvaud, J.-A., Hoshino, M., and Hirai, M.: Non-adiabatic ion acceleration in the Earth magnetotail and its various manifestations in the plasma sheet boundary layer, Space Sci. Rev., 164, 133-181, doi:10.1007/s11214-011-9858-9, 2011.

Grigorenko, E. E., Malova, H. V., Artemyev, A. V., Mingalev, O. V., Kronberg, E. A., Koleva, R., Daly, P. W., Cao, J. B., Sauvaud, J.-A., Owen, C. J., Zelenyi, L. M.: Current sheet structure and kinetic properties of plasma flows during a near-Earth magnetic reconnection under the presence of a guide field, J. Geophys. Res., 118, 3265-3287, doi:10.1002/jgra.50310, 2013.

Holland, D. L. and Chen, J.: On chaotic conductivity in the magnetotail, Geophys. Res. Lett., 19, 1231-1234, doi:10.1029/92GL01121, 1992.

Holland, D. L., Chen, J., and Agranov, A.: Effects of a constant cross-tail magnetic field on the particle dynamics in the magnetotail, J. Geophys. Res., 101, 24997-25002, doi:10.1029/95JA02282, 1996.

Horton, W.: Chaos and structures in the magnetosphere., Phys. Rep., 283, 265-302, doi:10.1016/S0370-1573(96)00063-4, 1997.

Horton, W. and Tajima, T.: Decay of correlations and the collisionless conductivity in the geomagnetic tail, Geophys. Res. Lett., 17, 123-126, doi:10.1029/GL017i002p00123, 1990.

Kan, J. R.: Tail-like reconfiguration of the plasma sheet during the substorm growth phase, Geophys. Res. Lett., 17, 2309-2312, doi:10.1029/GL017i013p02309, 1990.

Karimabadi, H., Pritchett, P. L., and Coroniti, F. V.: Particle orbits in two-dimensional equilibrium models for the magnetotail, J. Geophys. Res., 95, 17153-17166, doi:10.1029/JA095iA10p17153, 1990.

Karimabadi, H., Daughton, W., and Quest, K. B.: Physics of saturation of collisionless tearing mode as a function of guide field, J. Geophys. Res., 110, 3214, doi:10.1029/2004JA010749, 2005.

Kaufmann, R. L., Lu, C., and Larson, D. J.: Cross-tail current, fieldaligned current, and By, J. Geophys. Res., 99, 11277-11296, doi:10.1029/94JA00490, 1994.

Kuznetsova, M. M. and Roth, M.: Thresholds for magnetic percolation through the magnetopause current layer in asymmetrical magnetic fiels, J. Geophys. Res., 100, 155-174, doi:10.1029/94JA02329, 1995.
Landau, L. D. and Lifshitz, E. M.: Vol. 1: Mechanics, Course of Theoretical Physics, Pergamon Press, 1st Edn., 1960.

Le, A., Egedal, J., Ohia, O., Daughton, W., Karimabadi, H. and Lukin, V. S.: Regimes of the Electron Diffusion Region in Magnetic Reconnection, Phys. Rev. Lett., 110, 125004, doi:10.1103/PhysRevLett.110.135004, 2013.

Liou, K. and Newell, P. T.: On the azimuthal location of auroral breakup: Hemispheric asymmetry, Geophys. Res. Lett., 37, L23103, doi:10.1029/2010GL045537, 2010.

Litvinenko, Y. E.: Particle acceleration in reconnecting current sheets with a nonzero magnetic field, Astrophys. J., 462, 997, doi:10.1086/177213, 1996.

Litvinenko, Y. E.: Particle acceleration by magnetic reconnection, in: Energy conversion and particle acceleration in the solar corona, edited by: Klein, L., vol. 612 of Lecture Notes in Physics, Berlin Springer Verlag, 213-229, 2003.

Lukianova, R. Y., Kozlovskii, A., and Christiansen, F.: Fieldaligned currents in the winter and summer hemispheres caused by IMF By, Geomagnetism and Aeronomy/Geomagnetizm i Aeronomiia, 52, 300-308, doi:10.1134/S0016793212020089, 2012.

Malova, H. V., Popov, V. Y., Mingalev, O. V., Mingalev, I. V., Mel'nik, M. N., Artemyev, A. V., Petrukovich, A. A., Delcourt, D. C., Shen, C., and Zelenyi, L. M.: Thin current sheets in the presence of a guiding magnetic field in Earth's magnetosphere, J. Geophys. Res., 117, A04212, doi:10.1029/2011JA017359, 2012.

Masuda, S., Kosugi, T., and Hudson, H. S.: A hard X-ray tworibbon flare observed with Yohkoh/HXT, Solar Phys., 204, 5567, doi:10.1023/A:1014230629731, 2001.

Neishtadt, A. I.: Change of an adiabatic invariant at a separatrix, Soviet J. Plasma Phys., 12, 568-573, 1986.

Neishtadt, A.: On the change in the adiabatic invariant on crossing a separatrix in systems with two degrees of freedom, J. Appl. Math. Mech., 51, 586-592, doi:10.1016/0021-8928(87)90006-2, 1987.

Neishtadt, A. and Vasiliev, A.: Phase change between separatrix crossings in slow-fast Hamiltonian systems, Nonlinearity, 18, 1393-1406, doi:10.1088/0951-7715/18/3/023, 2005.

Northrop, T. G.: The adiabatic motion of charged particles, Interscience Publishers John Wiley and Sons, New York-LondonSydney, 1963.

Numata, R. and Yoshida, Z.: Chaos-induced resistivity in collisionless magnetic reconnection, Phys. Rev. Lett., 88, 045003, doi:10.1103/PhysRevLett.88.045003, 2002.

Østgaard, N. and Laundal, K. M.: Auroral asymmetries in the conjugate Hemispheres and interhemispheric currents, Washington DC American Geophysical Union Geophysical Monograph Series, 197, 99-111, doi:10.1029/2011GM001190, 2012.

Parker, E. N.: Spontaneous current sheets in magnetic fields: with applications to stellar x-rays, International Series in Astronomy and Astrophysics, Vol. 1, New York: Oxford University Press, 1994.

Paschmann, G., Haaland, S., and Treumann, R.: Auroral Plasma Physics, Space Sci. Rev., 103, 1-19, doi:10.1023/A:1023030716698, 2002.

Pellat, R. and Schmidt, G.: Absence of particle drift in magnetic fields of translational symmetry, Phys. Fluids, 22, 381-382, doi:10.1063/1.862591, 1979. 
Petrukovich, A. A.: Dipole tilt effects in plasma sheet By: statistical model and extreme values, Ann. Geophys., 27, 1343-1352, doi:10.5194/angeo-27-1343-2009, 2009.

Petrukovich, A. A.: Origins of plasma sheet $B_{y}$, J. Geophys. Res., 116, A07217, doi:10.1029/2010JA016386, 2011.

Pitaevskii, L. P. and Lifshitz, E. M.: Vol. 10: Physical Kinetics, Course of Theoretical Physics, New York, Pergamon Press, 1981.

Rong, Z. J., Wan, W. X., Shen, C., Li, X., Dunlop, M. W., Petrukovich, A. A., Hau, L.-N., Zhang, T. L., Rème, H., Du, A. M., and Lucek, E.: Profile of strong magnetic field $\mathrm{B}_{y}$ component in magnetotail current sheets, J. Geophys. Res., 117, A06216, doi:10.1029/2011JA017402, 2012.

Roth, M., de Keyser, J., and Kuznetsova, M. M.: Vlasov theory of the equilibrium structure of tangential discontinuities in space plasmas, Space Sci. Rev., 76, 251-317, doi:10.1007/BF00197842, 1996.

Schrijver, C. J.: Driving major solar flares and eruptions: A review, Adv. Space Res., 43, 739-755, doi:10.1016/j.asr.2008.11.004, 2009.

Silin, I. and Büchner, J.: Nonlinear instability of thin current sheets in antiparallel and guided magnetic fields, Phys. Plasmas, 10, 3561-3570, doi:10.1063/1.1599357, 2003.

Sinitsyn, A., Dulov, E., and Vedenyapin, V.: Kinetic Boltzmann, Vlasov and related Equations, Elsevier, 2011.

Sitnov, M. I., Zelenyi, L. M., Malova, H. V., and Sharma, A. S.: Thin current sheet embedded within a thicker plasma sheet: Selfconsistent kinetic theory, J. Geophys. Res., 105, 13029-13044, doi:10.1029/1999JA000431, 2000.

Sivukhin, D. V.: Motion of charged particles in electromagnetic fields in the drift approximation, vol. 1, 1-104, Consultants Bureau, New York, 1965.

Syrovatskii, S. I.: Key aspects of flare theory, Akademiia Nauk SSSR Izvestiia Seriia Fizicheskaia, 43, 695-707, 1979.

Timofeev, A. V.: On the problem of constancy of the adiabatic invariant during change of the type of motion, Sov. JETP, 75, 13031308, 1978.

Ukhorskiy, A. Y., Sitnov, M. I., Millan, R. M., and Kress, B. T.: The role of drift orbit bifurcations in energization and loss of electrons in the outer radiation belt, J. Geophys. Res., 116, A09208, doi:10.1029/2011JA016623, 2011.

Vainchtein, D. L., Büchner, J., Neishtadt, A. I., and Zelenyi, L. M.: Quasiadiabatic description of nonlinear particle dynamics in typical magnetotail configurations, Nonlin. Processes Geophys., 12, 101-115, doi:10.5194/npg-12-101-2005, 2005.

Vainshtein, D. L., Zelenyi, L. M., Neishtadt, A. I., and Savenkov, B. V.: Jumps in an adiabatic invariant with small initial values, Plasma Physics Reports, 25, 299-303, 1999.

Whipple, E. C., Hill, J. R., and Nichols, J. D.: Magnetopause structure and the question of particle accessibility, J. Geophys. Res., 89, 1508-1516, doi:10.1029/JA089iA03p01508, 1984.
Wing, S., Newell, P. T., Sibeck, D. G., and Baker, K. B.: A large statistical study of the entry of interplanetary magnetic field Ycomponent into the magnetosphere, Geophys. Res. Lett., 22, 2083-2086, doi:10.1029/95GL02261, 1995.

Wisdom, J.: A perturbative treatment of motion near the 3/1 commensurability, Icarus, 63, 272-289, doi:10.1016/00191035(85)90011-9, 1985.

Zelenyi, L. M., Sitnov, M. I., Malova, H. V., and Sharma, A. S.: Thin and superthin ion current sheets. Quasi-adiabatic and nonadiabatic models, Nonlin. Processes Geophys., 7, 127-139, doi:10.5194/npg-7-127-2000, 2000.

Zelenyi, L. M., Delcourt, D. C., Malova, H. V., and Sharma, A. S.: "Aging" of the magnetotail thin current sheets, Geophys. Res. Lett., 29, 1608, doi:10.1029/2001GL013789, 2002a.

Zelenyi, L. M., Delcourt, D. C., Malova, H. V., Sharma, A. S., Popov, V. Y., and Bykov, A. A.: Forced current sheets in the Earth's magnetotail: Their role and evolution due to nonadiabatic particle scattering, Adv. Space Res., 30, 1629-1638, doi:10.1016/S0273-1177(02)00427-1, 2002b.

Zelenyi, L. M., Dolgonosov, M. S., Bykov, A. A., Popov, V. Y., and Malova, K. V.: Influence of trapped plasma on the structure of collisionless thin current sheets, Cosmic Research, 40, 357-366, 2002c.

Zelenyi, L. M., Malova, H. V., and Popov, V. Y.: Splitting of thin current sheets in the Earth's magnetosphere, Sov. JETP Lett., 78, 296-299, doi:10.1134/1.1625728, 2003.

Zelenyi, L. M., Dolgonosov, M. S., Grigorenko, E. E., and Sauvaud, J.: Universal properties of the nonadiabatic acceleration of ions in current sheets, Sov. JETP Lett., 85, 187-193, doi:10.1134/S0021364007040017, 2007.

Zelenyi, L. M., Malova, H. V., Artemyev, A. V., Popov, V. Y., and Petrukovich, A. A.: Thin current sheets in collisionless plasma: Equilibrium structure, plasma instabilities, and particle acceleration, Plasma Physics Reports, 37, 118-160, doi:10.1134/S1063780X1102005X, 2011.

Zelenyi, L. M., Neishtadt, A. I., Artemyev, A. V., Vainchtein, D. L., and Malova, H. V.: Quasiadiabatic dynamics of charged particles in a space plasma, Physics Uspekhi, 56, 347-394, doi:10.3367/UFNe.0183.201304b.0365, 2013.

Zhou, X.-Z., Ge, Y. S., Angelopoulos, V., Runov, A., Liang, J., Xing, X., Raeder, J., and Zong, Q.-G.: Dipolarization fronts and associated auroral activities: 2. Acceleration of ions and their subsequent behavior, J. Geophys. Res., 117, A10227, doi:10.1029/2012JA017677, 2012.

Zhu, Z. and Parks, G.: Particle orbits in model current sheet with a nonzero B(y) component, J. Geophys. Res., 98, 7603-7608, doi:10.1029/92JA02366, 1993. 